\title{
Meteorología mesoescalar en Marte
}

\author{
Jorge PLA-GARCÍA ${ }^{1,2,3}$ y Scot C.R. RAFKIN ${ }^{2}$ \\ ${ }^{1}$ Centro de Astrobiología, Torrejón de Ardoz, 28850, España, jpla@cab.inta-csic.es \\ ${ }^{2}$ Southwest Research Institute, Boulder, CO, 80302, Estados Unidos \\ ${ }^{3}$ Space Science Institute, Boulder, CO, 80301, Estados Unidos
}

Recibido: 22/04/2016

Aceptado: 26/09/2016

\section{Resumen}

El auge de la exploración marciana a partir de la década de los noventa, junto con la optimización de los modelos meteorológicos terrestres de área limitada, ha propiciado el escenario ideal para la adaptación de este tipo de modelos a la atmósfera de Marte. Su uso se ha convertido en una parte fundamental para la interpretación de los datos generados por las misiones espaciales al planeta rojo y para proporcionar las restricciones de sus condiciones ambientales. Además se han convertido en una herramienta fundamental para la evaluación de riesgos en la etapa de entrada, descenso y aterrizaje de las misiones al planeta rojo. Las circulaciones de mesoescala en Marte que contribuyen notablemente a la estructura y dinámica de la atmósfera son los vientos de ladera, los procesos de inyección de polvo atmosférico y los procesos de transporte de volátiles en la capa límite atmosférica. En este artículo se incluye también a modo de ejemplo de modelización mesoescalar de Marte la simulación meteorológica con el modelo MRAMS del cráter Gale donde el rover Curiosity de la misión Mars Science Laboratory (MSL) de la NASA aterrizó en agosto de 2012 con la estación meteorológica española REMS a bordo, la cual servirá además para validar el modelo. Las interpretaciones meteorológicas revelan un entorno meteorológico dinámicamente muy complejo. Dentro del cráter se producen circulaciones atmosféricas, fruto de la suma no lineal de las escalas local, regional y sinóptica. Se pueden distinguir claramente dos periodos atmosféricos en el cráter: periodo semiaislado, durante gran parte del año marciano (Ls 0, 90 y 180) y periodo de mezcla, durante el verano (Ls 270). En el periodo semiaislado, un flujo de masas de aire pasa por encima del cráter y genera una capa límite muy estable, los vientos son de componente sur y las masas de aire en el suelo del cráter son generalmente más frías que la del exterior, por ello, los vientos de laderas descendentes, de origen termodinámico, generalmente no se introducen en el cráter, por lo que el nivel de mezcla es muy bajo. Sin embargo durante el verano (Ls 270) los vientos son los más fuertes de todo el año, sobre todo durante la noche, siendo generalmente de componente norte. Esta estación (Ls 270) es única en el cráter, ya que las masas de aire interiores se mezclan con la atmósfera externa debido a los fortísimos flujos del norte que inundan completamente el cráter por procesos dinámicos conocidos como ondas de montaña.

Palabras clave: Atmósfera de Marte; Dinámica atmosférica; Clima de Marte; Estructura atmosférica.

\section{Mars mesoscale meteorology}

\begin{abstract}
The revitalizing of Mars exploration in the early 1990s combined with the maturation of terrestrial mesoscale modeling codes provided fertile ground for the application of suitably modified models to the atmosphere of Mars. The use of mesoscale models, particular for Mars, has become an integral part of interpreting the data returned from missions and providing constraints and bounds on environmental conditions in support of mission planning and operations. Mesoscale models are ideal tools for greatly minimize risk during entry, descent, and landing phases. Slope winds, dust injection processes and atmospheric volatiles transport are the main Mars mesoscale circulations that affect the atmospheric
\end{abstract}


structure and dynamics. In this article is also included as an example the meteorological simulation of Gale Crater environment, in which the Mars Science Laboratory (MSL) Rover Curiosity landed in August 2012, with the spanish weather station Rover Environmental Monitoring Station (REMS) aboard. The observational data record provides sufficient means against which to evaluate mesoscale model. Meteorological interpretations show a dynamically complex environment. Local, regional and synoptic scales all interact in nonlinear ways to produce the observed weather. Two different atmospheric periods could be distinguished inside the crater: semi-isolated period during most of the martian year (Ls 0, 90 and 180) and mixing period during the summer (Ls 270). During the semi-isolated period, warm air from south overrides the crater creating a very stable boundary layer. Crater air mass is generally much colder than outside the crater forcing downslope flows, thermodynamically driven, to not scour crater and hence decreasing mixing level in this period. Ls 270 was shown to be an anomalous season when air within and outside the crater was well mixed by strong, flushing, northerly flow and large amplitude breaking mountain waves. At other seasons, the air in the crater was more isolated form the surrounding environment.

Key words: Mars atmosphere, Atmospheric dynamics, Mars climate, Atmosopheric structure.

Sumario: Introducción 1. Modelado mesoescalar en Marte 1.1. Modelo mesoescalar MRAMS 2. Descripción del experimento para el cráter Gale 2.1. Inicialización y condiciones de contorno 2.2. Tiempo de integración y frecuencia de salida 3. Comparación de las observaciones frente a las simulaciones 3.1. Datos de presión 3.2. Datos de temperatura del suelo 3.3. Datos de temperatura del aire 4. Interpretación meteorológica 4.1. Circulación de escala global o planetaria 4.2. Circulaciones regionales 4.3. Vientos de ladera y ondas de montaña 4.4. Capa Limite Atmosférica (CLA) debilitada 5. Conclusiones. Agradecimientos. Referencias.

\section{Normalized Reference}

Pla-Garcia J., Rafkin S.C.R., (2016). Meteorología mesoescalar en Marte, Física de la Tierra., Vol., 28, 129-161.

\section{Introducción}

Marte presenta muchos de los fenómenos meteorológicos que se producen en la Tierra como la circulación atmosférica meridional (Haberle et al., 1993), estaciones asociadas a la oblicuidad planetaria, perturbaciones baroclínicas y frentes en superficie (Tyler y Barnes, 2005), nubes de condensación de vapor de agua y dióxido de carbono (Michaels et al., 2006), circulaciones diurnas catabáticas y anabáticas (Rafkin et al., 2002; Spiga 2011b), tormentas de polvo (Cantor et al., 2001) y remolinos de polvo conocidos como dust devils (Fisher et al. 2005). Sin embargo las diferencias con la Tierra, como la menor irradiación solar, la baja densidad atmosférica, la compleja topografía y la alta variabilidad en la cantidad de aerosoles (compuestos principalmente por polvo radiativamente activo), deben tenerse en cuenta a la hora de modelar numéricamente la atmósfera de Marte.

De todos los planetas del Sistema Solar, Marte es el que presenta una mayor similitud con las condiciones ambientales terrestres, ya que está compuesto por roca y metales de alta densidad, además de rotar aproximadamente al mismo ritmo y en el mismo sentido que la Tierra. Un día marciano, denominado "sol", dura 24 horas y 20 minutos, y su año se prolonga durante 668,59 soles (dos años terrestres), motivo por el cual las estaciones en Marte tienen aproximadamente el doble de duración que las terrestres. El eje de rotación de Marte es además muy parecido al de la Tierra, con 
una inclinación de $25^{\circ}$ (el de la Tierra es de $23,5^{\circ}$ ), pero con una excentricidad de su órbita alrededor del Sol mucho más grande $(0,093$, mientras que en la Tierra es de $0,017)$ lo cual produce una gran diferencia en la irradiación solar máxima recibida en el hemisferio norte comparada con el sur. Como consecuencia de este diferente aporte energético, las estaciones resultan más extremas en el hemisferio sur. Una muestra de ellos es que las temperaturas veraniegas en el hemisferio sur pueden ser hasta $30 \mathrm{~K}$ más calientes que las del verano del hemisferio norte.

La longitud solar (Ls) es el ángulo que forma el planeta Marte con el Sol, medido desde el equinoccio de primavera del hemisferio norte, donde Ls es 0 . La longitud solar Ls 90 corresponde al solsticio de verano, Ls 180 corresponde al equinoccio de otoño y Ls 270 al solsticio de invierno, todos ellos relativos al hemisferio norte.

El forzamiento radiativo del ambiente de Marte es un elemento clave en el modelado atmosférico. Aunque la atmósfera está compuesta casi en su totalidad de $\mathrm{CO}_{2}$, la presión media global en superficie es de tan solo $\sim 6 \mathrm{mb}$ (apenas un $1 \%$ de la presión terrestre) dando como resultado una constante de tiempo radiativa pequeña (inferior a un sol) y un debilitamiento del calentamiento por efecto invernadero (Gierasch and Goody, 1968). Esta tenue atmósfera, junto con la ausencia de masas oceánicas y la baja inercia térmica del suelo marciano, producen una gran amplitud térmica diaria. Además, Marte está situado a una distancia del Sol 1,52 veces más lejana que la Tierra, recibiendo solo un $43 \%$ de la luz solar que recibe nuestro planeta.

A diferencia de la capa atmosférica terrestre cercana a la superficie, la cual es calentada principalmente por flujos turbulentos, la de Marte es calentada por la convergencia del flujo radiativo (Michaels y Rafkin, 2004). Durante el día, la superficie caliente irradia un flujo de energía ascendente, donde es absorbida por el polvo y $\mathrm{CO}_{2}$ de las capas atmosféricas cercanas al suelo. Este proceso genera una capa limite atmosférica (CLA, también llamada capa limite planetaria o capa límite convectiva) en Marte muy profunda (unos 8-10 km), con un gradiente adiabático muy inestable (Smith et al., 2004). Por la noche este proceso se invierte, desarrollándose una fuerte inversión térmica (Spiga et al., 2011). El efecto del calentamiento radiativo se incrementa debido a la baja masa atmosférica: una unidad de energía entrante puede generar un incremento de temperatura casi 100 veces mayor que en la Tierra debido a la diferencia de densidad. Por esta misma razón, la eficiencia del transporte turbulento de energía se ve fuertemente debilitado en Marte, aunque las variaciones turbulentas pueden ser mucho más grandes que en la Tierra (Rafkin y Michaels, 2004; Haberle et al., 1993; Larsen et al 2002; Savijärvi and Kauhanen, 2008). Una de las principales consecuencias de la diferencia en el calentamiento atmosférico entre la Tierra y Marte es que la elevación del terreno en este último ejerce una influencia pequeña en las temperaturas cercanas a la superficie. Se puede decir que, mientras las isotermas tienden a ser cuasi-horizontales en la Tierra, las isotermas en Marte siguen la topografía. Esta diferencia en la estructura de la temperatura tiene fuertes implicaciones en la naturaleza de las circulaciones mesoescalares de los dos planetas. 
La diferencia de temperatura entre el suelo y la capa atmosférica superficial dependerá de la cantidad de radiación solar recibida, pudiendo llegar a tener $30^{\circ} \mathrm{C}$ de diferencia entre el suelo y la capa atmosférica superficial. Esta diferencia varía también entre localizaciones, dependiendo no solo de la latitud, sino también del albedo, la inercia térmica del suelo y de la cantidad de polvo atmosférico. Esta discrepancia sustancial en las temperaturas aire-suelo entre Marte y la Tierra deriva en una incorrecta interpretación de las temperaturas máximas marcianas en la literatura y webs de divulgación, en las que la temperatura del suelo a menudo se confunde con la del aire.

La temperatura media del aire en Marte cerca de la superficie es alrededor de 218 $\mathrm{K}\left(-55^{\circ} \mathrm{C}\right)$, la cual varía desde $\operatorname{los} 276,3 \mathrm{~K}\left(+3,15^{\circ} \mathrm{C}\right)$ de máxima en regiones cercanas al ecuador hasta los $170 \mathrm{~K}$ (por debajo de $-100^{\circ} \mathrm{C}$ ) de mínima en los casquetes polares. Las temperaturas nocturnas en los polos están limitadas por la temperatura de condensación del $\mathrm{CO}_{2}$, que es de $150 \mathrm{~K}\left(-123,15^{\circ} \mathrm{C}\right)$. Las temperaturas registradas por las diferentes misiones son accesibles desde la base de datos del Sistema de Datos Planetarios de NASA (http://pds.jpl.nasa.gov/).

La temperatura del aire más alta registrada en Marte fue medida por el instrumento REMS, que se encuentra el cráter Gale próximo al ecuador en el hemisferio sur marciano (Figura 1), justo después del mediodía del sol 754 en la longitud solar 199, momento en el que el cráter recibe mayor irradiación solar. Otras temperaturas máximas del aire cerca del ecuador marciano medidas a la misma altura sobre el suelo son las del aterrizador Viking 1 , con $255,75 \mathrm{~K}\left(-17,4{ }^{\circ} \mathrm{C}\right)$ en Ls 147 y el aterrizador Pathfinder, con $261,55 \mathrm{~K}\left(-11,6^{\circ} \mathrm{C}\right)$ en Ls 164 . Los rover Spirit y Opportunity, aunque carecían de instrumentación meteorológica, podían (y Opportunity sigue pudiendo) estimar indirectamente la temperatura del aire aplicando algoritmos de correlación con la banda de $15 \mu \mathrm{m}$ de $\mathrm{CO}_{2}$ usando el espectrómetro mini-TES, obteniendo los registros máximos de $260 \mathrm{~K}\left(-13.15^{\circ} \mathrm{C}\right)$ en el caso de Spirit y de $265 \mathrm{~K}\left(-8.15^{\circ} \mathrm{C}\right)$ en el caso de Opportunity (Spanovich et al., 2006). Por lo tanto, aunque la escasez de medidas meteorológicas en Marte hace que estas no sean globalmente representativas, se podría concluir que registrar temperaturas del aire en Marte positivas es algo excepcional.

El polvo atmosférico también tiene un importante impacto radiativo. Tanto la insolación como el flujo descendente infrarrojo se ven afectados por el contenido de polvo atmosférico. Generalmente, el polvo tiene un efecto anti-invernadero en las longitudes de onda solares, pero actúa con un efecto contrario en el infrarrojo. Por lo tanto, un aumento de la cantidad de polvo atmosférico reduce en superficie el flujo entrante solar pero aumenta sin embargo el flujo descendente infrarrojo. El resultado neto es una disminución en la amplitud de la señal diurna de la temperatura tanto del aire como del suelo, aumentando las temperaturas nocturnas y disminuyendo las diurnas (Haberle et al. 1982). El polvo atmosférico tiene una fuerte implicación en el clima y la circulación general de la atmósfera marciana y por ende en las operaciones de las misiones de exploración. Por desgracia, el conocimiento que tenemos sobre la dinámica de los dust devils en la CLA es bastante pobre. 


\section{Modelado mesoescalar en Marte}

Los modelos meteorológicos de área limitada aplicados a Marte son de especial importancia para el estudio de fenómenos horizontales de pequeña escala (aquellos menores de 100 kilómetros) como los vientos de ladera, los procesos de inyección de polvo atmosférico, los procesos de transporte de volátiles en la CLA o cuando se pretende simular las condiciones atmosféricas en la fase de entrada, descenso y aterrizaje de una misión y así poder diseñarla de forma adecuada. Debido a que los recursos computacionales se utilizan para realizar la resolución numérica de las ecuaciones que caracterizan los procesos atmosféricos en un área limitada es necesario utilizar condiciones de contorno. Estos modelos de área limitada permiten, a diferencia de los modelos de circulación general (MCG), aumentar la resolución horizontal y vertical de la simulación en igualdad de recursos computacionales, a cambio de no calcular las condiciones de contorno atmosféricas alrededor de los bordes de su dominio. Estas condiciones de contorno pueden ser introducidas de forma arbitraria o bien suministradas mediante las salidas de las simulaciones de un MCG. El desarrollo de modelos meteorológicos de área limitada para Marte requiere la aplicación de conocimientos de meteorología terrestre, en donde los modelos numéricos de predicción meteorológica se acoplan dentro de los MCG. El modelado mesoescalar permite realizar predicciones meteorológicas a corto plazo y en escalas locales. Los MCG son necesarios por tanto para calcular las condiciones de contorno y predecir cambios en escalas temporales más largas. Uno de los rasgos más importantes de los modelos de área limitada, junto a su mayor resolución, es que no necesitan balance hidrostático para poder permitir la mezcla convectiva vertical (Rafkin et al., 2001; Michaels y Rafkin, 2003). Los modelos de área limitada pueden resolver fenómenos microescalares inferiores a 100 metros de resolución entre puntos, aunque no pueden resolver movimientos turbulentos, motivo por el cual estos tienen que ser parametrizados. Aún con esta alta resolución, existen fenómenos meteorológicos que se producen en escalas menores al del tamaño de la malla. La oportunidad de modelar con resoluciones varios órdenes de magnitud mayor que los MCG abre la puerta a una representación más explícita de algunos procesos que operan dentro del espaciado de malla de un MCG. El uso de modelos de área limitada para aumentar la resolución puede ayudar en el calibrado, o al menos para identificar sus debilidades, de algunos esquemas de parametrización de los MCG.

En la Tierra, los modelos meteorológicos de área limitada son modelos numéricos tridimensionales de predicción meteorológica con una resolución horizontal y vertical suficientemente alta como para predecir fenómenos atmosféricos de mesoescala y microescala. Estos fenómenos son a menudo producidos por la topografía o están relacionados con procesos convectivos. Es importante recalcar que para Marte los modelos de área limitada no son modelos predictivos ya que utilizan siempre como entrada los resultados de un MCG en un procedimiento conocido como downscaling 
dinámico, a diferencia de los terrestres que normalmente son inicializados con observaciones reales (proceso conocido como reanálisis), aunque también pueden arrancar en frío o utilizar downscaling dinámico.

Las imágenes obtenidas por orbitadores y robots en superficie, tanto aterrizadores como robot exploradores, muestran claramente como la atmósfera marciana es bastante activa en la meso y la microescala. Algunas de las rachas de viento son de una escala tan pequeña que se pueden clasificar como circulaciones microescalares producidas por la interacción del viento con algún obstáculo topográfico. Uno de los rasgos más dinámicamente activos observados en Marte son los dust devils. Estos remolinos juegan un papel fundamental en el mantenimiento de la cantidad de polvo atmosférico (Newman et al., 2002; Kahre et al., 2006; Basu et al., 2004).

Dado que muchos de los fenómenos atmosféricos que se producen en Marte son de carácter micro y mesoescalar, y que estos tienen un papel protagonista en la circulación general (por ejemplo en lo que se refiere al ciclo del polvo), es importante el desarrollo, mantenimiento y mejora de los modelos de área limitada marcianos capaces de simular estos fenómenos.

Los fenómenos atmosféricos de mesoescala se clasifican dentro del dominio espacio-temporal entre los sistemas de microescala de corta duración y los sistemas de escala sinóptica (escala global) de larga duración. En la práctica, la división entre microescala y mesoescala, y mesoescala y escala sinóptica es bastante difusa. Aunque muchos de los fenómenos atmosféricos considerados típicamente mesoescalares han sido observados por imágenes orbitales y en menor medida mediante teledetección y mediciones in situ, son los modelos numéricos combinados con la información derivada de análogos terrestres las herramientas más eficientes a la hora de estudiar los flujos térmicos de pendiente, las ondas de gravedad, las tormentas de polvo y las circulaciones que producen las nubes de hielo ( de $\mathrm{CO}_{2}$ y de $\mathrm{H}_{2} \mathrm{O}$ ), considerados todos ellos fenómenos mesoescalares.

Las circulaciones mesoescalares en Marte tienen una influencia muy grande en la estructura y dinámica de la atmósfera. En muchos casos, las circulaciones son mucho más intensas y generalizadas que en la Tierra. Por ejemplo, se piensa que los flujos térmicos de ladera dominan la meteorología local en todo el planeta Marte excepto en las regiones más llanas. En la Tierra sin embargo dichos flujos solo son predominantes donde el forzamiento sinóptico es pequeño y la topografía es muy accidentada. En Marte, la estructura y dinámica de la atmósfera está íntimamente relacionada con las variaciones de la topografía, incluso con las relativamente pequeñas.

El uso de modelos de área limitada para Marte se ha convertido en una parte fundamental en la interpretación de los datos obtenidos por las misiones enviadas al planeta rojo. Además, los modelos se están utilizando cada vez más para proporcionar detalles de las condiciones ambientales necesarios para la planificación y operación de las misiones (Rafkin et al., 2003; Togio et al., 2003; Vasavada et al., 2012; Chen et al., 2010). 
En términos de hidrostática, el gradiente de presión horizontal producido por los gradientes de temperatura es mucho más grande en Marte que en la Tierra y por ende los flujos térmicos de ladera en el planeta rojo son unas 2,5 veces más fuertes (Rafkin y Michaels, 2003; Ye et al., 1990). Es por ello que las técnicas utilizadas para el planeta Tierra, en las que los modelos mesoescalares son inicializados desde MCG extrapolando datos atmosféricos globales a mesoescalares a través del gradiente adiabático, no funcionan para el planeta Marte. En cambio se utilizan otras técnicas que conservan la estructura térmica vertical cerca de la superficie asegurando que no se introduzcan gradientes adiabáticos inestables ni gradientes de presión horizontales espurios entre columnas adyacentes (Tyler et al., 2002).

Tradicionalmente muchos de los resultados del modelado mesoescalar de Marte no se podían validar directamente y se utilizaba para ello las imágenes y datos térmicos obtenidos de forma remota. Por ejemplo los vientos calentados adiabáticamente que forman parte de los flujos descendentes de ladera, se identificaban estudiando la señal térmica de superficie observada desde órbita (Spiga, 2011) y los rastros dejados por el polvo o la alineación de las dunas proporcionan datos sobre la dirección de la viento.

Uno de los éxitos más importantes hasta la fecha del modelado mesoescalar de Marte ha sido la satisfactoria simulación de las condiciones atmosféricas marcianas sin la necesidad de reanálisis ni de datos observacionales en la inicialización del modelo o en la definición de las condiciones de contorno. A cambio, estas simulaciones han utilizado como entrada las salidas de los MCG de Marte, los cuales a su vez tampoco han sido inicializados con datos derivados del estado de la atmósfera. Estos MCG arrancan desde un estado de reposo con una atmósfera isoterma. Los datos de salida de los MCG se consideran datos climáticos, no meteorológicos, ya que se refieren al tiempo meteorológico promedio sobre un periodo tradicional de años o décadas.

\subsection{Modelo mesoescalar MRAMS}

MRAMS (Mars Regional Atmospheric Modeling System, Rafkin et al., 2001) es un modelo meteorológico de área limitada adaptado al planeta Marte. Simula las circulaciones atmosféricas marcianas a escala regional y local. MRAMS es una adaptación de RAMS (Regional Atmospheric Modeling System), un modelo meteorológico de área limitada no hidrostático diseñado simular la meteorología terrestre (Pielke et al., 1992). RAMS, ampliamente utilizado para pronosticar el clima, fue diseñado para simular a escala sinóptica, mesoescala y microescala, los flujos atmosféricos sobre una topografía compleja, y la simulación de sistemas atmosféricos sobre terreno complejo. MRAMS está explícitamente diseñado para simular circulaciones atmosféricas en Marte a nivel de mesoescala y microescala con propiedades superficiales de alta resolución. Entre sus principales características incluye un modelo dinámico no hidrostático con ecuaciones para el movimiento vertical, polvo atmosférico y vapor de agua, un modelo de física atmosférica para el hielo de dióxido de carbono y un modelo de regolito que incluye deposición y sublimación de $\mathrm{CO}_{2}$. 
Varios proyectos de exploración de Marte de la NASA, incluyendo los Mars Exploration Rovers (Spirit y Oportunity) (Rafkin et al., 2003), el aterrizador Phoenix (Tamppari et al., 2008), el rover Curiosity (MSL) (Vasavada 2012; Chen et al., 2010), el aterrizador InSight y el rover Mars2020, han utilizado MRAMS para estudiar una amplia variedad de circulaciones atmosféricas.

\section{Descripción del experimento para el cráter Gale}

Para simular el entorno meteorológico de la misión MSL, el modelo MRAMS ha sido configurado con siete mallas anidadas centradas sobre la zona de aterrizaje dentro del cráter Gale (Figura 1).
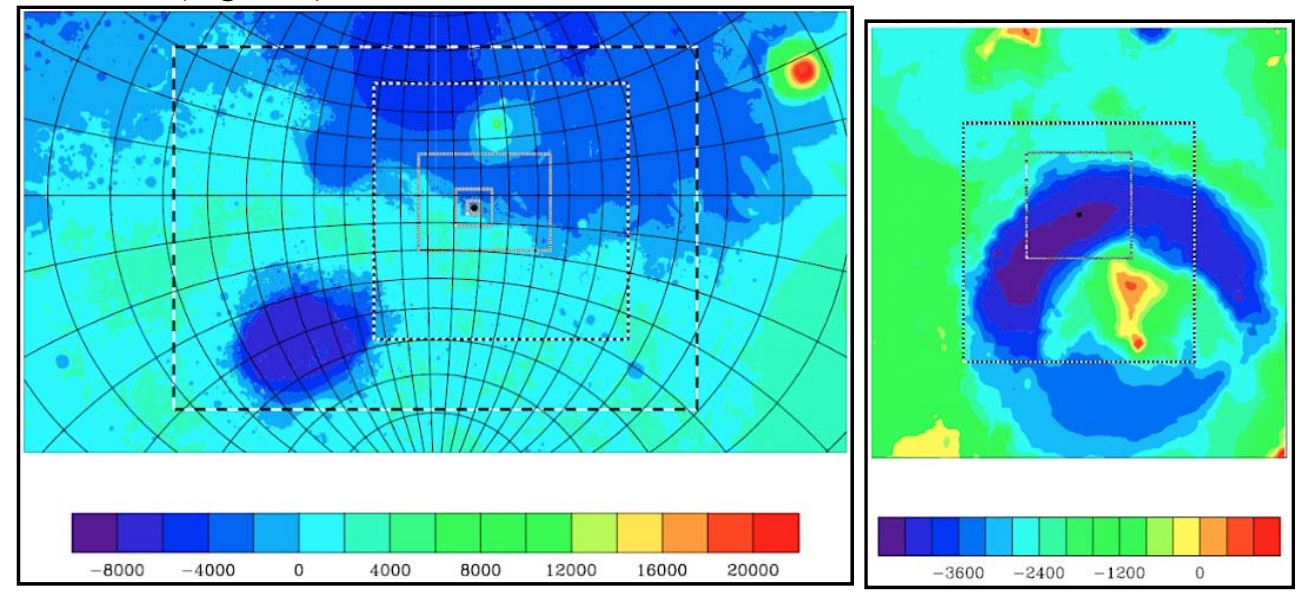

Figura 1. Espaciado de malla horizontal para las mallas 1 a 7 (izquierda) y 4 a 7 (derecha), centradas sobre la zona de aterrizaje de MSL dentro del cráter Gale. El espaciado en cada malla se representa en la figura con una línea discontinua blanca y negra. La elevación de la topografía se muestra con un código de colores obtenido del instrumento MOLA.

Las mallas fueron configuradas, en la medida de lo posible, para incluir en ellas aquellas regiones topográficas que pudieran influir en el experimento. La malla más externa y de menor resolución (malla 1) cubre gran parte de los hemisferios norte y sur, incluyendo la cuenca de impacto Hellas y la dicotomía hemisférica. Esta configuración captura los fuertes flujos topográficos que se producen en las cercanías de la dicotomía hemisférica así como los flujos estacionales de la circulación general meridional (células de Hadley) los cuales tienen dimensiones casi globales. Las mallas han sido diseñadas para minimizar en la medida de lo posible que accidentes topográficos importantes se encontraran dentro de los límites de una malla. El espaciado horizontal (distancia entre puntos) es de 240, 80, 26.7, 8.9, 2.96, 0.98 y 0.33 kilómetros de la malla 1 a la 7 respectivamente. Todas las mallas tienen la misma 
configuración vertical. El nivel más bajo, en el que se pronostican la temperatura del aire y la presión, es de $\sim 14$ metros sobre el suelo. Este espaciado vertical ha sido configurado para tener una separación creciente entre niveles, alcanzando un máximo de 2500 metros en los niveles más altos. Los niveles verticales van cambiando gradualmente desde los niveles más superficiales que son paralelos a la topografía, a los completamente horizontales en la cima del modelo. El espaciado vertical no excede los 100 metros en el primer kilómetro de altura de la atmósfera, ni los 400 metros en los tres siguientes. La cima de la atmósfera en el modelo se sitúa a una altura de 51 kilómetros con 50 niveles verticales.

\subsection{Inicialización y condiciones de contorno}

Las salidas obtenidas con el MCG de NASA Ames (Kahre et al., 2006) se han utilizado para inicializar el estado de la atmósfera en MRAMS. Las condiciones de contorno han sido incluidas en MRAMS de forma análoga.

El polvo ha sido parametrizado utilizando los datos promediados zonalmente del instrumento TES (Thermal Emission Spectrometer del orbitador Mars Global Surveyor) en años sin tormentas globales y siguiendo un perfil vertical de tipo Conrath-Nu (Conrath, 1975). Este parámetro describe como varia la profundidad de la columna de polvo atmosférico con la latitud y las estaciones, siendo máxima en la latitud subsolar. El hielo de $\mathrm{CO}_{2}$ superficial ha sido parametrizado en función de los datos derivados del MCG de NASA Ames para la localización y momento de arranque del experimento. Las propiedades de la superficie marciana son inicializadas en MRAMS con los datos obtenidos por el instrumento TES para inercia térmica del suelo y albedo, y con los datos del instrumento MOLA para la topografía y la pendiente. MRAMS tiene en cuenta los efectos producidos por las sombras de la topografía y la radiación en pendiente. Los datos de la inercia térmica del suelo obtenidos por TES no tienen la resolución suficiente como para captar algunas de las variaciones del suelo dentro del cráter Gale. Durante el primer verano de la misión (Ls 270) la inercia térmica del suelo en la zona conocida como Yellowknife Bay era tan diferente a la observada desde órbita que se tuvo que parametrizar dentro del modelo a un valor de $460 \mathrm{SI}$, basado en el trabajo de Hamilton et al. 2014. Dado que los datos de inercia térmica utilizados en MRAMS son generalmente más bajos que los reales, se espera que las curvas diarias de temperatura del suelo sobreestimen la amplitud diurna, tal y como se describe en el apartado 3.2.

Algo similar sucede con los datos de albedo dentro del cráter. A diferencia de la inercia térmica, no se ha realizado ningún cambio del albedo en el modelo, por lo que se esperaría que las simulaciones fueran ligeramente más calientes de lo que deberían, como así es.

\subsection{Tiempo de integración y frecuencia de salida}

En el experimento se han simulado cuatro soles con una resolución de cinco mallas y posteriormente se han añadido tres soles más aumentando la resolución hasta siete 
mallas. El primer sol siempre se descarta, ya que se utiliza como adaptación del modelo. Todas las simulaciones empezaron poco antes del amanecer local. MRAMS no es un modelo global y no puede, en teoría, simular un año marciano. Las series de datos anuales obtenidas por REMS pueden estudiarse en detalle en el artículo de Martínez et al. (2016) de este mismo volumen. Para obtener las condiciones representativas de un año marciano se han realizado simulaciones en cuatro longitudes solares que coinciden con los solsticios y equinoccios, de forma que los resultados pueden ser comparados directamente con sus correspondientes soles de operación de REMS, cuyos datos han sido promediados cada cinco minutos.

\section{Comparación de las observaciones frente a las simulaciones}

Solo tenemos datos de cuatro experimentos meteorológicos en la superficie Marte: las estaciones meteorológicas de los aterrizadores Viking 1 y 2 (VL1 y VL2), el experimento de estructura atmosférica ASI/MET del aterrizador Mars Pathfinder y el instrumento REMS de la misión Mars Science Laboratory Curiosity rover (GómezElvira et al., 2012). Este último era el único que en el momento de la elaboración de este artículo seguía funcionando.

Una característica común de todos estos experimentos, excepto REMS, es que todas estaban ubicadas en zonas relativamente llanas y de topografía poco compleja. VL1 obtuvo datos durante casi un año marciano en zonas llanas de latitudes subtropicales del hemisferio norte, mientras que VL2 obtuvo datos durante dos años marcianos en zonas llanas de latitudes medias del hemisferio norte, incluyendo un periodo de tormenta de polvo global. La misión Pathfinder obtuvo datos durante 83 soles en una zona topográficamente similar a VL1. La misión Phoenix obtuvo datos en zonas llanas de latitudes altas del hemisferio norte. En cambio, la misión MSL se encuentra en una de las zonas topográficamente más complejas de todas las visitadas en Marte hasta la fecha. La meteorología dentro del cráter es también una de las más complejas, debido a que la topografía condiciona la circulación atmosférica cerca de la superficie. El lugar elegido para el aterrizaje es uno de los de menor elevación dentro del cráter, ubicado entre el borde norte y el monte central Sharp.

El objetivo de las comparaciones entre las simulaciones con MRAMS y las observaciones del instrumento REMS es establecer una medida de confianza tanto en los resultados del modelo como en los datos de la estación meteorológica.

\subsection{Datos de presión}

Como se ha mencionado anteriormente, en cada casquete polar existe por encima de la capa de hielo de agua una capa superficial de hielo de $\mathrm{CO}_{2}$, capa que se sublima totalmente en el verano boreal y parcialmente en el verano austral. El ciclo de insolación solar da lugar a un intercambio de $\mathrm{CO}_{2}$ entre casquetes. En el invierno del hemisferio norte el $\mathrm{CO}_{2}$ de la atmósfera se congela sobre el casquete polar norte, y se sublima del casquete del polo sur. En invierno del hemisferio sur (verano del hemisferio 
norte) el proceso se invierte, lo que significa que cada año alrededor de un tercio de la atmósfera se intercambia entre los casquetes. Este ciclo controla por tanto el contenido global atmosférico de $\mathrm{CO}_{2}$ y determina la presión media diaria en una determinada estación. La existencia de dos mínimos y dos máximos, unos relativos y otro absolutos, en la presión anual medida por REMS (Martinez et al. 2016, este mismo volumen) corresponden a la alta excentricidad de la órbita de Marte y a una distribución asimétrica en la cantidad de hielo de $\mathrm{CO}_{2}$ entre los casquetes polares.

La comparación entre los datos de presión diaria medidos por el instrumento REMS y los obtenidos en las simulaciones del modelo MRAMS se muestran en la Figura 2. Uno de los aspectos más importantes para comprender la meteorología del cráter Gale son las variaciones de presión sobre la media. Algunas de las características básicas de estas variaciones de presión son inmediatamente evidentes en la gráfica, donde se aprecia como la estructura diurna varía considerablemente a lo largo del año. El equinoccio de verano (Ls 270) tiene una estructura relativamente suave semejante a una onda sinusoidal.

En el resto de estaciones, se aprecia mucha más estructura en la señal con varias chepas y armónicos de orden superior, ausentes en Ls 270. Esta ausencia responde a una escasa contribución de las circulaciones inferiores a la escala de la marea térmica del ciclo de presión. En esta estación concreta, las circulaciones global y regional neutralizan la circulación local del cráter. En Ls0, 90 y 180, la circulación del cráter y la regional de la dicotomía están mejor definidas que en Ls 270. El resultado de una circulación más localizada y organizada es la presencia de estructuras de alta frecuencia en la señal de presión.

La circulación del cráter produce una exportación neta de aire durante el día, como resultado de los flujos de ventilación de pendiente ascendentes, y una importación neta de aire por la noche como consecuencia de un flujo de aire de pendiente descendente. Las perturbaciones diarias de la presión media son el resultado de la marea térmica (dominada por los modos diurno y semidiurno) y por las circulaciones regional y local.

Los resultados del modelo mostrados en la Figura 2 son compatibles con cada uno de los rasgos observados, lo que sugiere que el modelo funciona satisfactoriamente a la hora de capturar los procesos físicos, incluyendo todas las escalas de movimientos relevantes que actúan para producir las variaciones diarias. La amplitud y la fase de la marea diaria (definida por los momentos máximo y mínimo de presión) son generalmente bien capturadas en el modelo. 


\section{MRAMS Pressure vs. REMS Pressure}
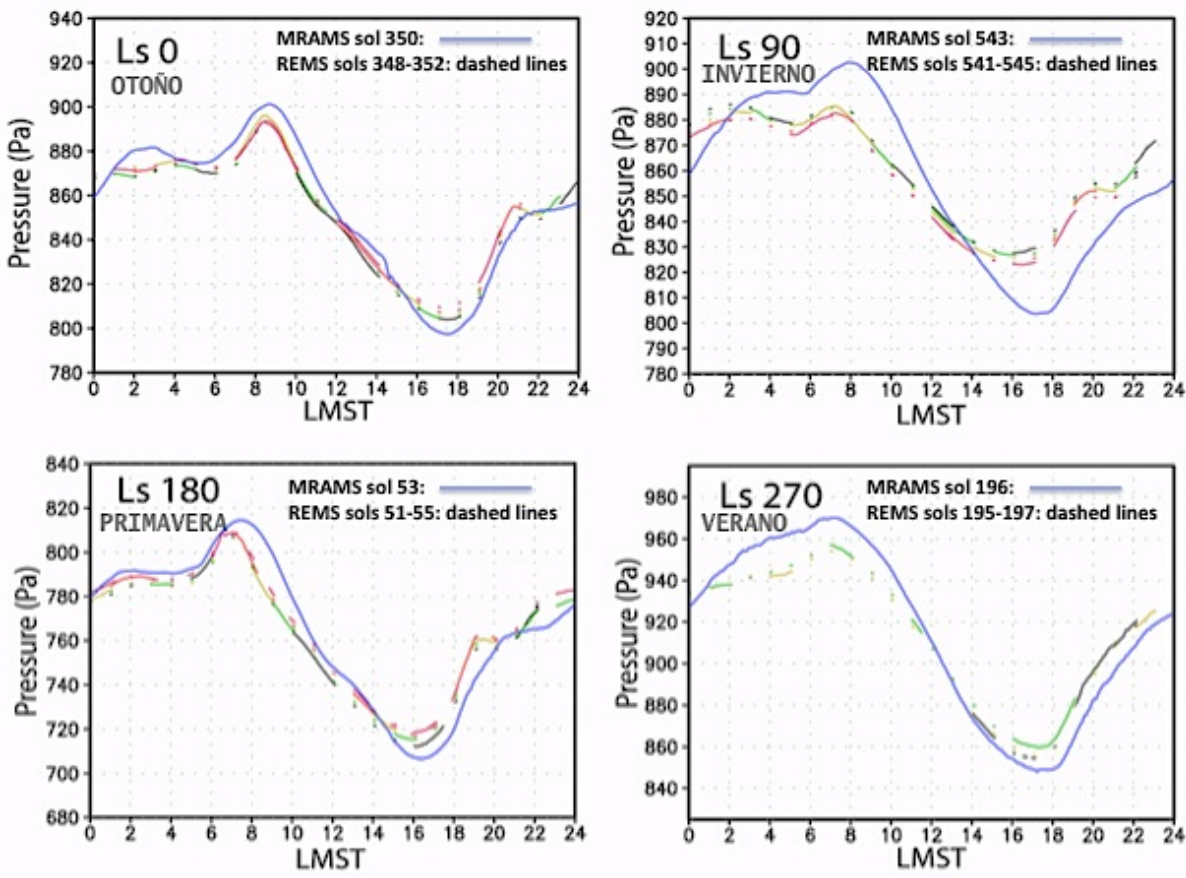

Figura 2. Señal diaria observada y modelada de presión en las cuatro estaciones. La línea continua es la señal del modelo MRAMS. Los datos de cinco soles consecutivos de REMS se representan en líneas discontinuas de diferentes colores. Adaptado de Pla-García et al. 2016.

Harri et al. (2014) encontraron una oscilación de presión en las horas vespertinas, aproximadamente entre las 1900 y las 2200 LMST del sol 48. Estas oscilaciones son de 0.5 Pa o incluso menores, con períodos de 12-18 minutos. Debido a que la frecuencia de salida del modelo es de 5 minutos, es difícil capturar ondas de período similar (se produce el efecto conocido como aliasing). Sin embargo, observando los resultados del modelo para Ls 180 (que equivale al Sol 53), se puede apreciar una oscilación similar comenzando aproximadamente a las 1900 LMST y disminuyendo alrededor de las 2200 LMST, momento en el que la curva de presión global comienza a subir rápidamente (Figura. 3). Estas oscilaciones están probablemente asociadas a una actividad de ondas de montaña, como se explicará en la sección de interpretación meteorológica. 

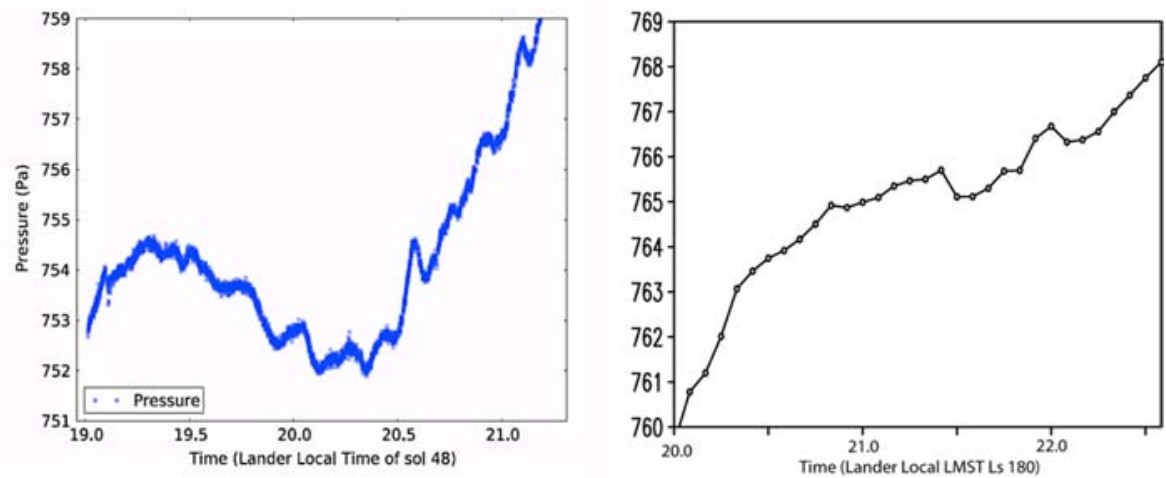

Figura 3. Oscilaciones de presión en el cráter Gale en Ls 180 (2000-2200LMST). Las observaciones están a la izquierda y los resultados del modelo a la derecha.

\subsection{Datos de temperatura del suelo}

Las comparaciones de temperatura del suelo se muestran en la Figura 4.

MRAMS Ground Temperature vs. REMS Ground Temperature
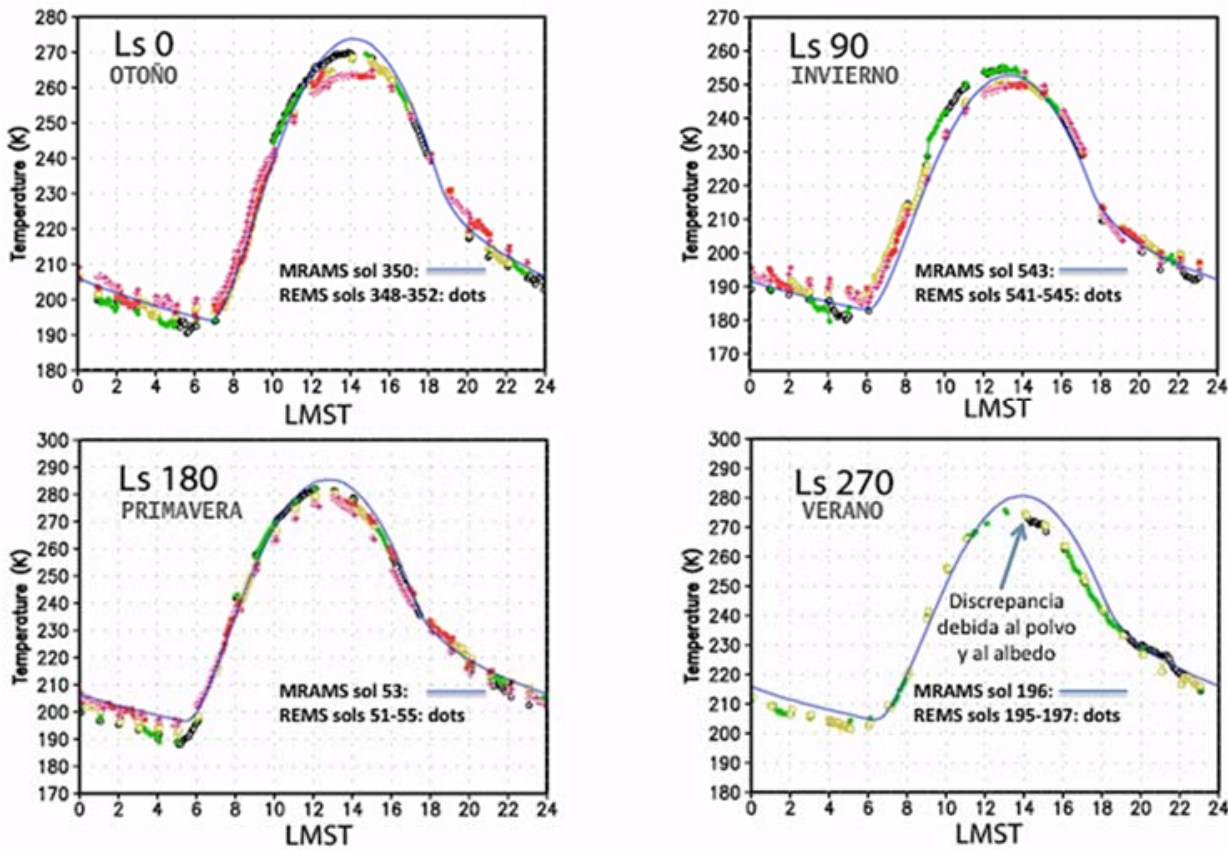

Figura 4. Señal diaria observada y modelada de temperatura del suelo en las cuatro estaciones. La línea continua es la señal del modelo MRAMS. Los datos de cinco soles consecutivos de REMS se representan con puntos de diferentes colores. Adaptado de Pla-García et al. 2016. 
REMS determina la temperatura del suelo a partir de la emisión infrarroja de la superficie, asumiendo una emisividad igual a uno. La temperatura del suelo es el diagnóstico del balance de energía de la superficie en el cual contribuye la insolación, los flujos infrarrojos descendentes (de la atmósfera al suelo) y ascendentes (del suelo a la atmósfera), la conducción en el subsuelo y el flujo atmosférico de calor turbulento. Un desequilibrio en alguno de estos componentes resultará en un cambio neto de la temperatura de la superficie y por lo tanto un cambio en la emisión infrarroja que puede ser detectada por el sensor de temperatura del suelo (GTS) de REMS. Las comparaciones de las simulaciones con los datos de REMS (Figura 4) muestran que la temperatura del suelo simulada está dentro de los márgenes de error de medición de $\sim 5 \mathrm{~K}$ en todas las estaciones, excepto en Ls 270. En Ls 0 y Ls 180, parece que la señal simulada es algo más caliente durante la tarde, aunque la temperatura está dentro de los márgenes de error de las observaciones. En Ls 90, el aumento de temperatura al amanecer es más rápido en las observaciones que en el modelo, aunque el resto de la señal de temperatura es coincidente con las observaciones. El modelo tiene en cuenta los efectos topográficos de las pendientes y de las sombras a la hora de realizar los cálculos de transferencia radiativa, pero se basa en la información obtenida a partir de la topografía de MOLA promediada para la resolución de la malla más interna del modelo (en este caso $333 \mathrm{~m}$ ). Un ascenso más rápido de la temperatura del suelo medida por el GTS podría ser el resultado de una orientación hacia el Sol más eficiente que la del modelo. Está pendiente tendría un impacto prácticamente despreciable durante el resto del día y ninguno durante la noche, donde el enfriamiento infrarrojo es el que domina.

En general, la buena representación en las simulaciones de las medidas observadas en Gale sugiere que el modelo ha sido inicializado con unos valores razonables de inercia térmica, albedo y contenido de polvo atmosférico en la mayoría de las estaciones. Teniendo en cuenta que los valores de albedo en el modelo están ligeramente subestimados, la tendencia de las simulaciones de tener una temperatura ligeramente superior a la observada en todas las estaciones es razonable, sobre todo en el verano (Ls 270). Esta mayor discordancia en Ls 270 comparada con las otras estaciones del año sugiere, en lugar de un error en la física del modelo, que los valores de albedo, inercia térmica o cantidad de polvo atmosférico no han sido inicializados de forma exacta. Si las parametrizaciones físicas fueran erróneas, entonces se esperarían desajustes en todas las estaciones, pero no es el caso. Una posibilidad es que el valor del albedo sea demasiado bajo en el modelo. En Ls 270, el rover se encontraba en la zona denominada Yellowknife Bay, donde el albedo podría haber alcanzado valores de 0,3, mientras que el valor utilizado en MRAMS (derivado de TES) es significativamente menor $(0,23)$. Un ajuste en el valor del albedo cambiaría las temperaturas del suelo en Ls 270 (incluidas en la Figura 4), para parecerse más a las observadas, sin embargo la fase de las curvas de temperatura no se vería afectada. 
Se puede apreciar una curiosa protuberancia en la curva de temperatura observada por REMS entre las 2000 y las 2200 LMST en Ls 270 que no parece apreciarse en las otras estaciones y que podría deberse a un evento de calentamiento rápido asociado con el paso de ondas de montaña. Hay que resaltar que la amplitud de la perturbación observada es menor que la incertidumbre de las mediciones.

Otra posible explicación podría ser el aumento de la turbulencia impulsado por el incremento de la cizalladura del viento en la inversión nocturna. A medida que la capa de inversión nocturna se desarrolla, los vientos por encima de esta se desacoplan de la superficie y la disminución de la fricción produce una aceleración neta. En ese momento, las inestabilidades de la cizalladura pueden empujar el aire cálido en altura hasta la superficie, produciendo una mezcla en las masas de aire.

\subsection{Datos de temperatura del aire}
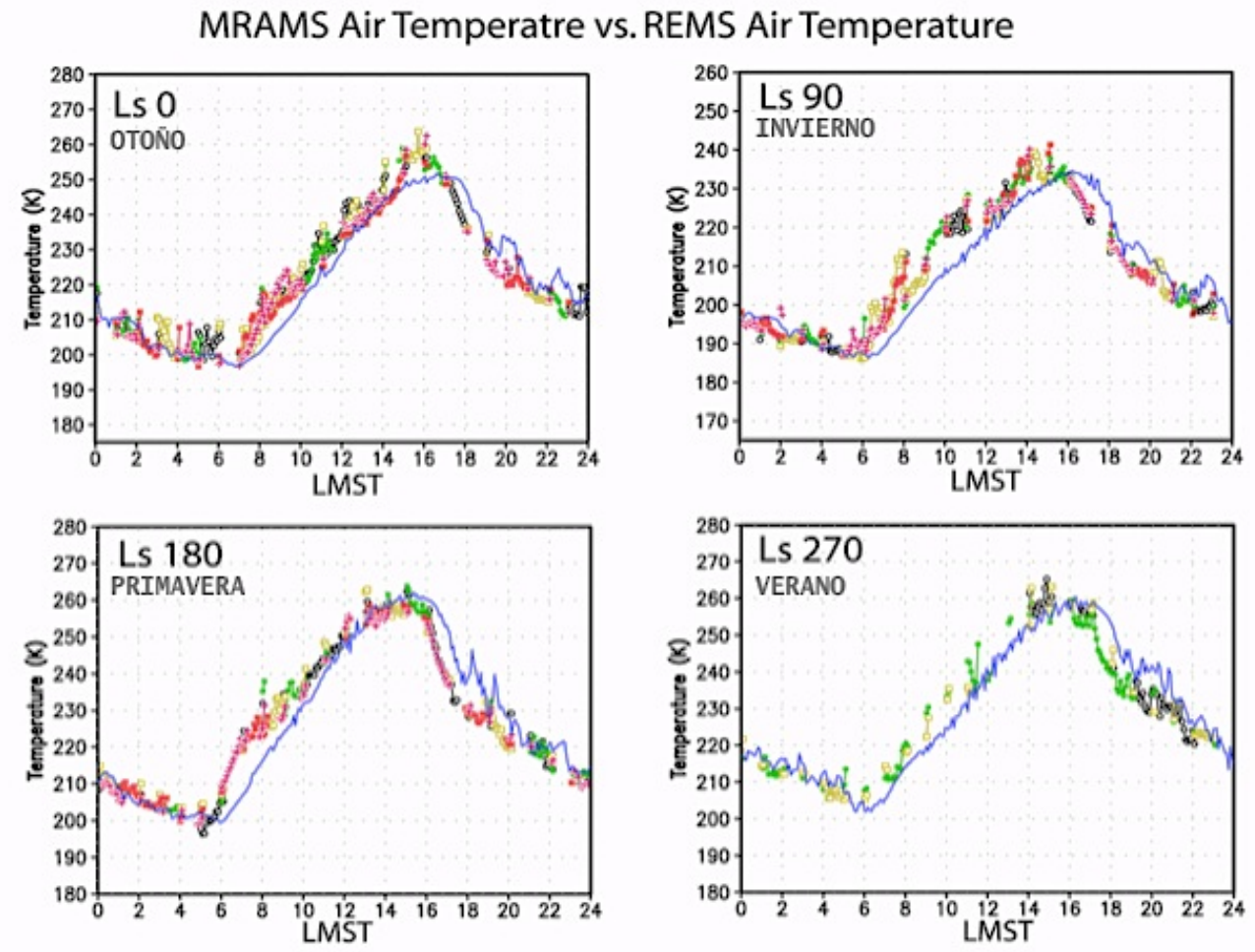

Figura 5. Igual que la figura 4 pero para la temperatura del aire, la cual está medida a 1.5 metros por encima del nivel del suelo. El nivel atmosférico más bajo ha sido simulado con MRAMS a una altura de $\sim 14 \mathrm{~m}$. 
Las comparaciones de temperatura del aire se muestran en la Figura 5. Es importante recalcar que la temperatura del aire observada se mide a una altura de $\sim 1,5 \mathrm{~m}$ sobre el nivel del suelo (AGL), mientras que MRAMS proporciona temperatura del aire en el nivel más bajo del modelo, el cual se encuentra a unos $\sim 14 \mathrm{~m}$. Como era de esperar, esta diferencia de altura hace que los valores de MRAMS sean más fríos que los observados por la mañana y al comienzo de la tarde, siendo más calientes el resto de la tarde y durante la noche. Este fenómeno se debe al gradiente superadiabático vespertino y a la fuerte inversión térmica nocturna (Schofield et al., 1997).

La mayor diferencia entre el modelo y las observaciones es el calentamiento excesivo de las temperaturas simuladas por la tarde, pero es difícil cuantificar cuánto es debido a la diferencia de altitud entre modelo y sensor. Una cierta cantidad de discrepancia puede no ser atribuible a la altitud, dado que las temperaturas de superficie del modelo también son un poco más calientes que las observadas.

Las temperaturas observadas muestran una gran variación por la tarde, durante los periodos de calentamiento solar, mientras que el modelo es relativamente suave durante ese mismo intervalo. Esto era de esperar, debido a que estas variaciones térmicas son debidas a la turbulencia de la CLA, las cuales no pueden ser resueltas en el modelo debido a que tienen un tamaño menor al del espaciado de malla de mayor resolución (las únicas turbulencias que el modelo puede resolver son los flujos turbulentos de mesoescala nocturnos, como las ondas de gravedad, porque están en una escala comparable o mayor que el espacio de malla). Sin embargo, el efecto submalla de la turbulencia es capturado por el modelo a través de la ecuación de pronóstico denominada energía cinética turbulenta (TKE).

El modelo muestra un pico de TKE durante la tarde (Figura 6), que es consistente con las variaciones de alta frecuencia observadas. Por la noche, el modelo resuelve las variaciones térmicas, las cuales son consistentes con las observaciones. Por lo tanto, las variaciones observadas no se relacionan con la turbulencia de pequeña escala (que no puede resolver el modelo), pero si con los flujos descendentes altamente variables y capturados por el modelo (los cuales son producidos dinámicamente como se explicará más adelante). 


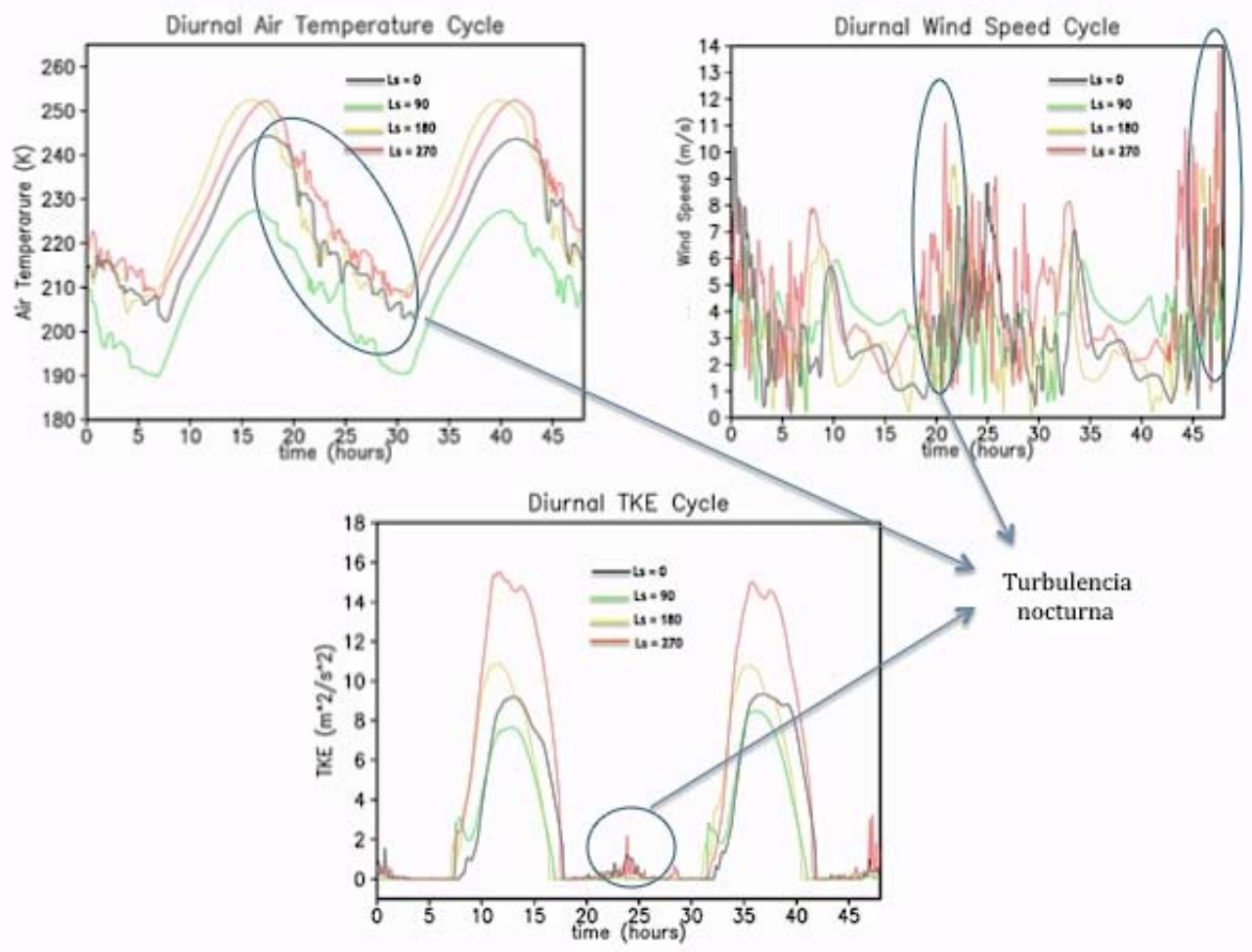

Figura 6. Turbulencia nocturna en el cráter Gale obtenida con MRAMS.

\section{Interpretación meteorológica}

El tiempo meteorológico observado en el cráter Gale puede ser considerado como la suma de los efectos derivados de las circulaciones atmosféricas en el contexto de las diferentes escalas (sinóptica, regional y local, Figura 7), tal y como se concluye en las interpretaciones extraídas de las simulaciones. En la escala global (sinóptica), la circulación meridional es producida por el gradiente de presiones a gran escala alimentado por el correspondiente gradiente térmico meridional. El forzamiento solar produce la fuerte marea térmica diaria que también interacciona con la topografía. Si bajamos a la escala regional, la dicotomía topográfica (tierras bajas al norte y altas al sur), las variaciones topográficas de escala continental y los grandes cráteres de impacto (como la cuenca Hellas) exhiben circulaciones térmicas de ladera que oscilan diariamente de forma ascendente y descendente. A nivel local, cráteres, valles y colinas producen de forma similar circulaciones térmicas directas que producen vientos de ladera ascendentes durante el día y descendentes durante la noche. Final- 
mente, en la microescala, se produce turbulencia convectiva y vórtices (incluyendo dust devils) que producen perturbaciones de alta frecuencia.

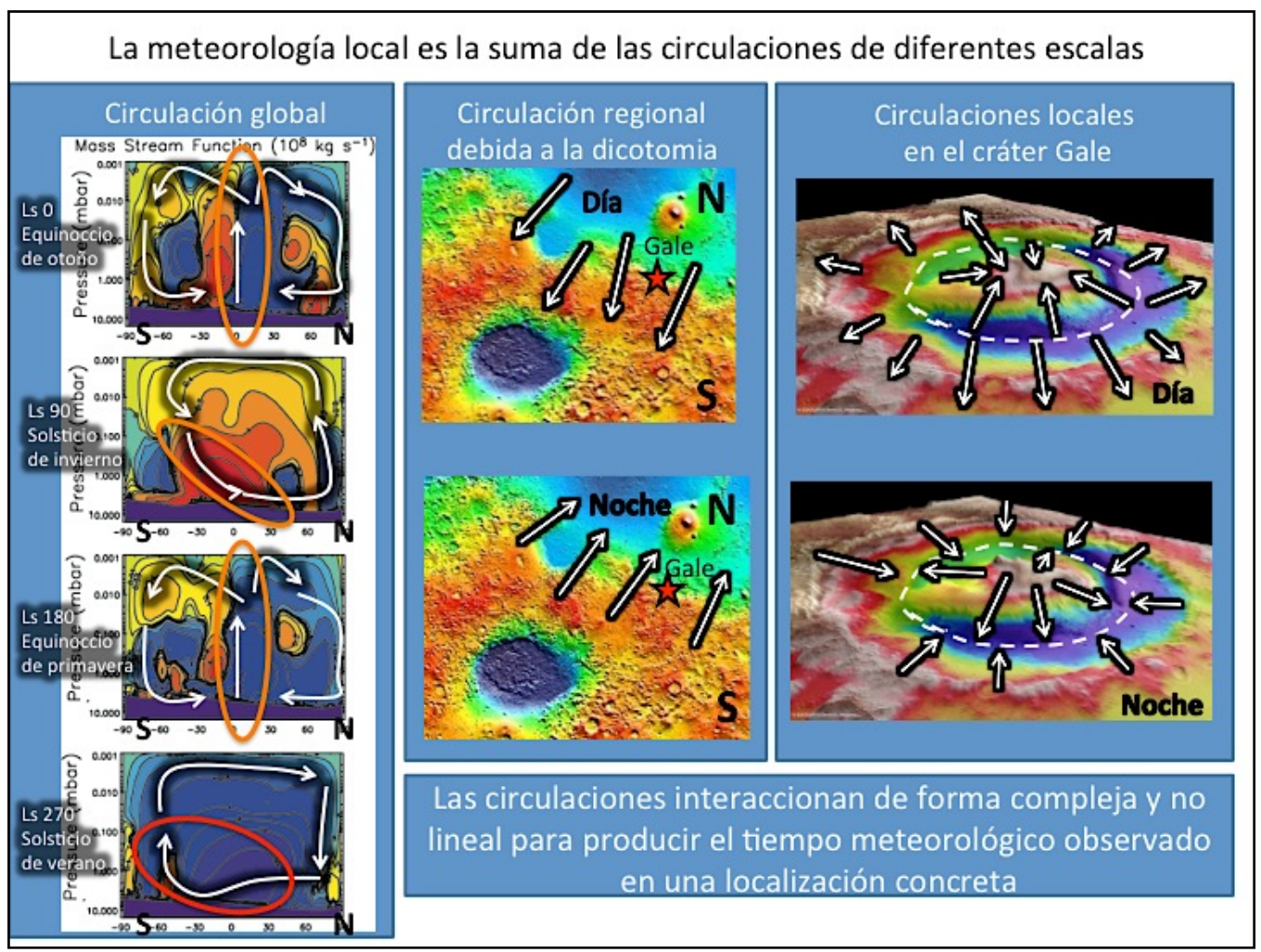

Figura 7. Escenario multiescalar. El cráter Gale se encentra cerca del ecuador marciano.

Todas estas circulaciones no suelen sumarse de forma lineal. Por ejemplo, una fuerte circulación asociada al cráter puede afectar sustancialmente la altura de la CLA dentro del mismo, y esto puede conducir a una modulación importante de la turbulencia y los vórtices convectivos. Del mismo modo, fuertes circulaciones de escala regional o global pueden alterar de forma considerable la circulación del cráter.

En el caso específico de Gale, las interacciones no lineales entre escalas son evidentes tanto en los resultados de MRAMS como en las observaciones de REMS.

\subsection{Circulación de escala global o planetaria}

Los cambios de estación producen en Marte cambios en la circulación meridional global, compuesta por una sola célula de Hadley (a diferencia de las tres terrestres, Hadley, Ferrel y Polar) que es de carácter global y cambia de signo al llegar el verano. Esto es algo que está bien establecido a través de los MCG de Marte, cuyos resultados 
son consistentes con los campos de temperatura globales derivados desde órbita (Haberle et al., 1993).

Esta célula de Hadley tiene una rama ascendente en las latitudes altas del hemisferio verano (Ls 90 para el HN y Ls 270 para el HS) y una rama de subsidencia en el hemisferio de invierno (Ls 270 para el HN y Ls 90 para el HS). Los vientos en superficie asociados a esta circulación fluyen desde el hemisferio de invierno al hemisferio de verano. Se produce así mismo un flujo de retorno en capas altas inverso al flujo de superficie (Figura 8).

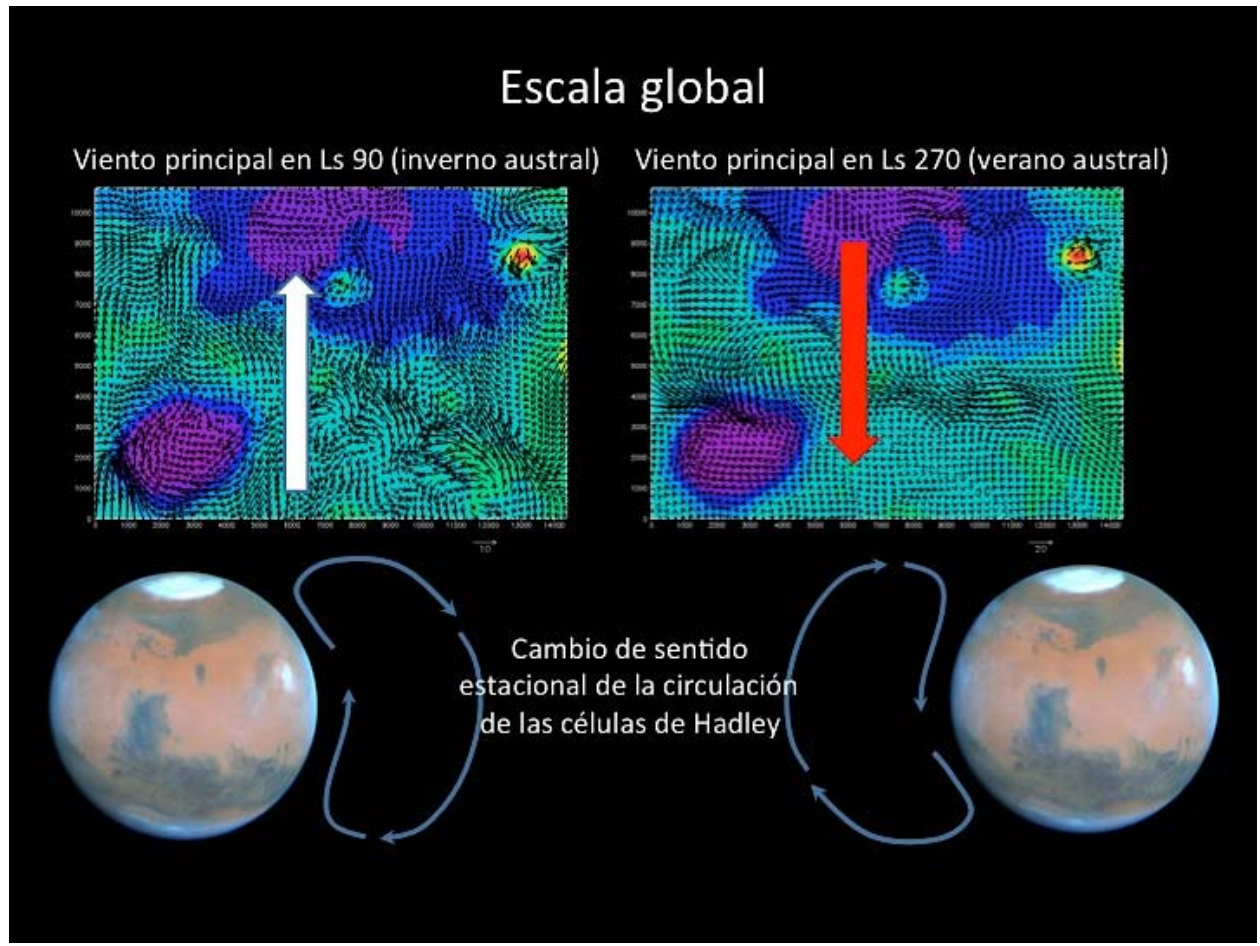

Figura 8. Malla 1 de MRAMS 240km (arriba). Células de Hadley (abajo).

Alrededor de los equinoccios hay un breve período de transición donde la rama ascendente cruza rápidamente de un hemisferio al otro, a medida que migra hacia su ubicación solsticial (Figura 7, lado izquierdo). Durante esta transición, hay una convergencia en la rama ascendente (similar a la zona de convergencia intertropical en la Tierra), generándose dos células de Hadley, una en cada hemisferio. Debido a la circulación global, los vientos sinópticos en superficie en la ubicación de Gale se invierten con el cambio de estación, teniendo vientos de componente norte en el equinoccio de verano (Ls 270) y de componente sur en el de invierno (Ls 90). En los solsticios, los vientos sinópticos podrían ir en cualquier dirección dado que la rama 
ascendente transita a través de la zona ecuatorial, por eso los denominamos neutros (Figura 7, lado izquierdo).

A diferencia de otras estaciones, en Ls 270 hay en promedio un flujo muy fuerte de componente norte en la región del cráter Gale, independientemente de la hora del día. En la escala global, el hemisferio sur está más caliente y atrae a los vientos del norte debido al gradiente de presión a gran escala producido. Por último, el calentamiento vespertino de la dicotomía tenderá a producir un flujo de pendiente ascendente que reforzará aún más los vientos del norte.

Por lo tanto, durante la tarde en Ls 270, la gran escala y la escala regional favorecen claramente los vientos del norte. Es de esperar que la dicotomía fuerce también el flujo de vientos descendentes de ladera durante la noche, debido a que estos vientos fríos son forzados termodinámicamente a descender. Sin embargo, lo que se aprecia es que este flujo sigue siendo de componente norte. Al parecer, la circulación meridional global es lo suficientemente fuerte como para contrarrestar el forzamiento topográfico de la dicotomía. En la escala global, hay un flujo muy fuerte de norte a sur sobre la región del cráter Gale en Ls 270 durante todos los momentos del día. Como se mostrará a continuación, la mezcla constructiva de todas las circulaciones de las diferentes escalas en Ls 270, hará que este periodo sea único en el cráter Gale.

\subsection{Circulaciones regionales}

Aunque existe gran complejidad meteorológica en la escala del cráter, se pueden asumir algunas generalidades. En todas las escalas hay una tendencia de los vientos a soplar pendiente arriba durante el día y hacia abajo por la noche. En el caso del cráter Gale, esto se traduce en una divergencia de los vientos del suelo del cráter, los cuales se desplazan hacia el monte Sharp y los bordes del cráter durante el día, realizando el recorrido inverso durante la noche, lo que les hace converger en el suelo del cráter (Figura 7 a la derecha).

La esencia de esta danza diaria se puede apreciar en todas las estaciones, sin embargo la fuerza de los vientos de pendiente varía considerablemente en función de la estación debido a las interacciones no lineales con los flujos de mayor escala. Además, las inestabilidades dinámicas mesoescalares (sobre todo las ondas de montaña y las ondas de gravedad de gran amplitud) también participan en la mezcla. Estas ondas, a diferencia de todas las circulaciones mencionadas con anterioridad, no son producidas por procesos termodinámicos, en lugar de ello, algunos flujos mesoescalares como las ondas de montaña se generan dinámicamente por la interacción del viento con la topografía.

Estos fenómenos dinámicos, que no son de origen catabático, pueden oponerse a las fuerzas de flotabilidad y proporcionar un mecanismo para forzar que las masas de aire caliente desciendan (cuando en condiciones normales deberían ascender por tener menor densidad) y que las masas de aire frío asciendan. Hacia las 1600h (LMST), el flujo ascendente de la dicotomía está completamente desarrollado y los vientos son de componente norte en todas las estaciones, además en el equinoccio de verano (Ls 270) 
la circulación meridional global refuerza los vientos ascendentes por la dicotomía (Figura 9).
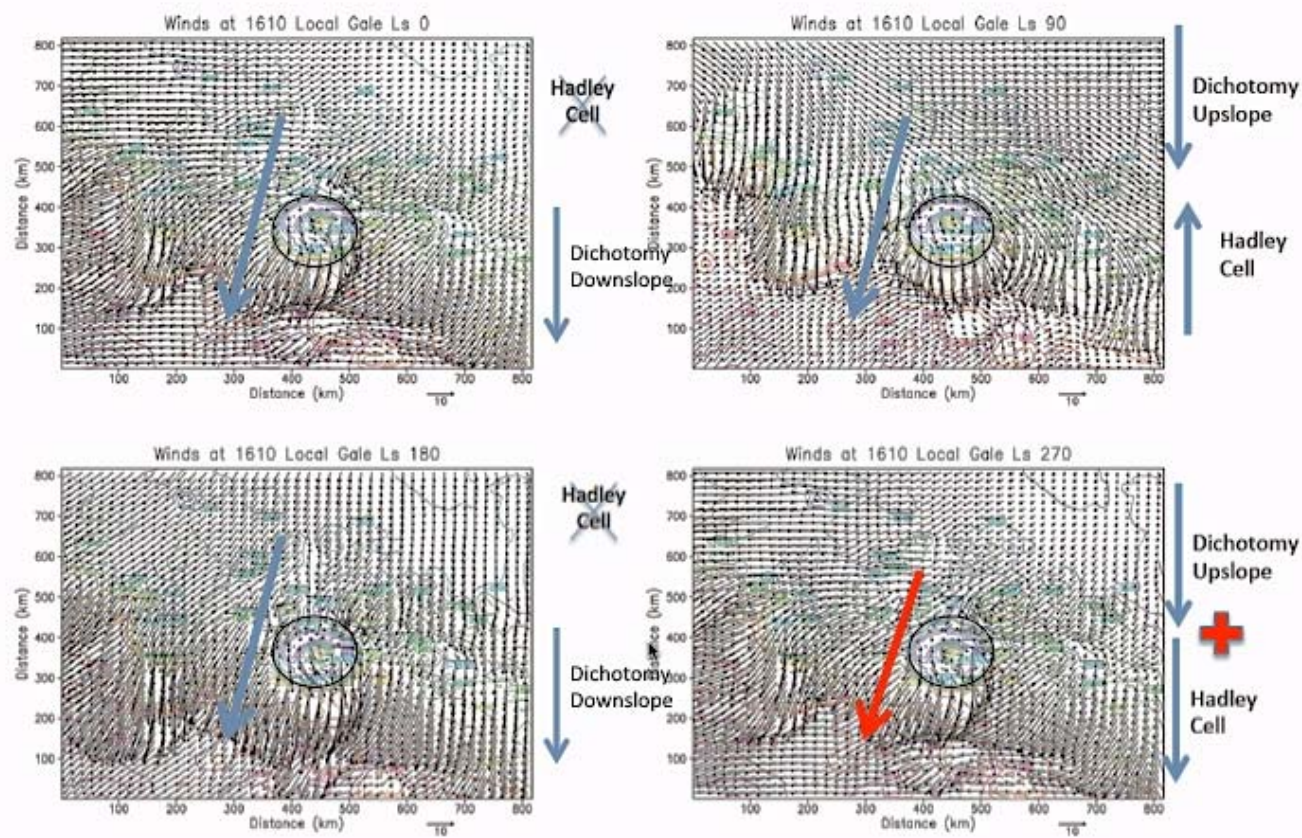

Figura 9. Escala regional (malla 4, 9km res.) durante el día (1610 LMST). Se aprecia una gran influencia de la topografía de la dicotomía en todas las estaciones. Vientos de componente norte durante el día. Desviación de vientos alrededor y sobre el cráter.

En Ls 0, 90 y 180, la temperatura potencial de las masas de aire del suelo del cráter son más frías que las del exterior del mismo, tal y como se aprecia en las diferencias de las propiedades térmicas de las mismas (Figuras 10 y 11). Esta situación térmica tiende a suprimir los movimientos verticales. 

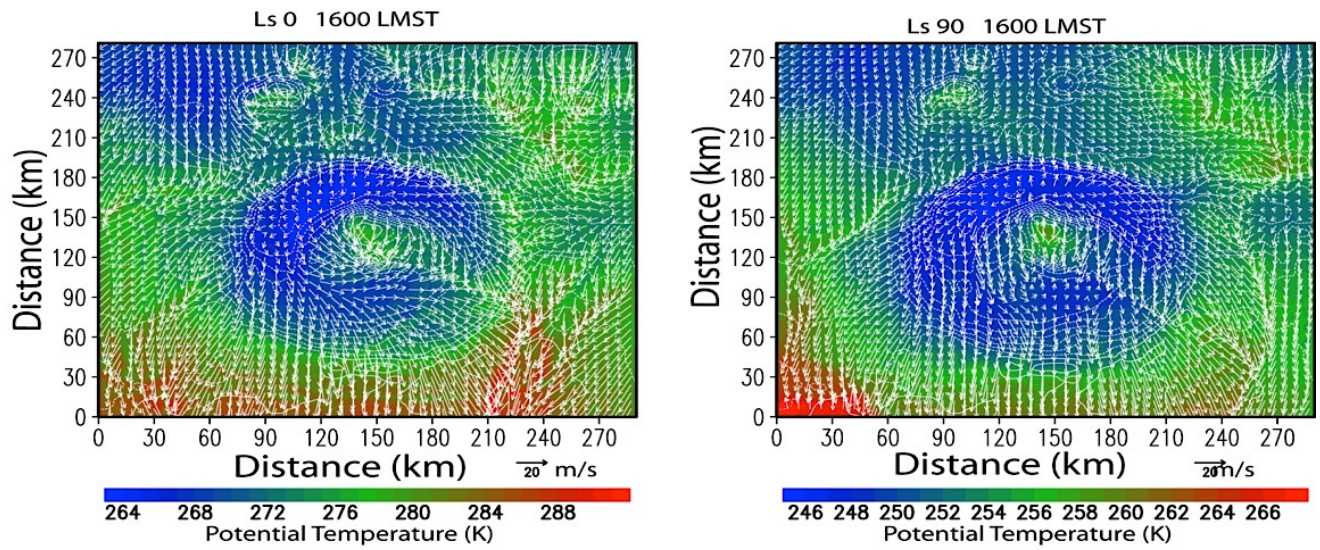

246248250252254256258260262264266
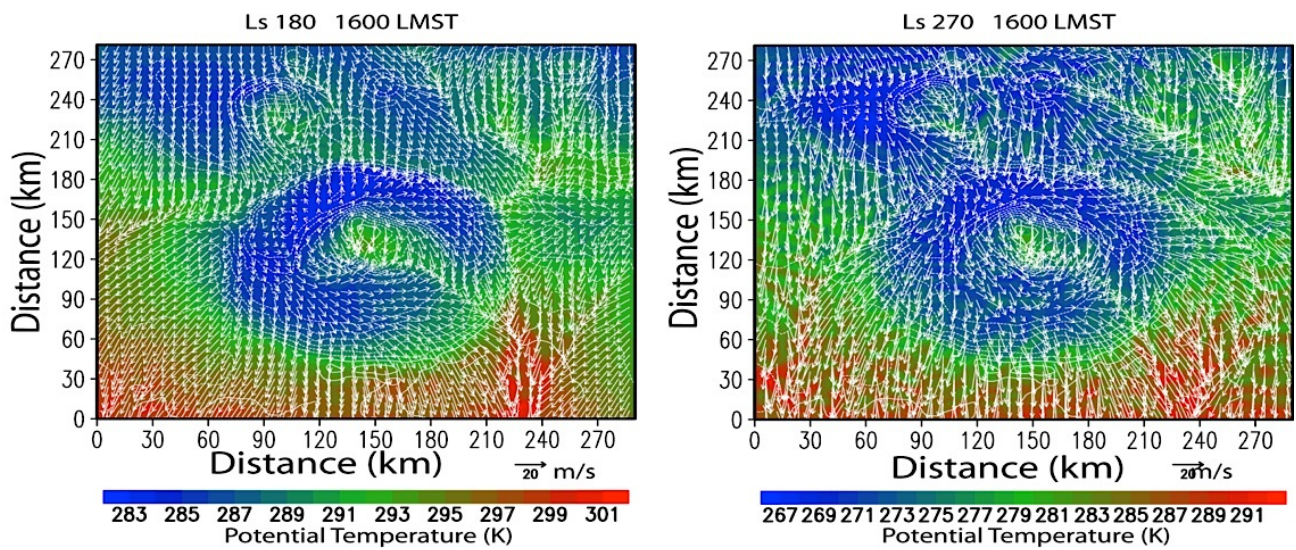

267269271273275277279281283285287289291 Potential Temperature $(\mathrm{K})$

Figura 10. Dirección y velocidad del viento en superficie (vectores) y temperatura potencial (colores) a las 1600 LMST en función de la estación (malla 5) en el cráter Gale. La escala del vector de viento se muestra en la parte inferior derecha de la imagen. Figura obtenida de Rafkin et al. 2016.

Con excepción de Ls 270, en general durante el año marciano el aire fuera del cráter es más cálido que el del interior. Cualquier masa de aire que descienda por los bordes del cráter hacia el interior tiende a rebotar, debido a principios de flotabilidad, y a no mezclarse con el aire más frío inferior.

Al final de la tarde (2200 LMST), el flujo ascendente por las laderas del cráter cambia a sentido descendente en todas las estaciones (Figura 11). La temperatura potencial elevada en las masas de aire del suelo del cráter en Ls 270 proviene de aire en altura transportado por la fuerte mezcla vertical asociada a ondas de montaña, las cuales, junto a los flujos descendentes de ladera, mantienen la atmósfera en los niveles bajos más cálida de lo que se esperaría de los procesos radiativos y convectivos. 


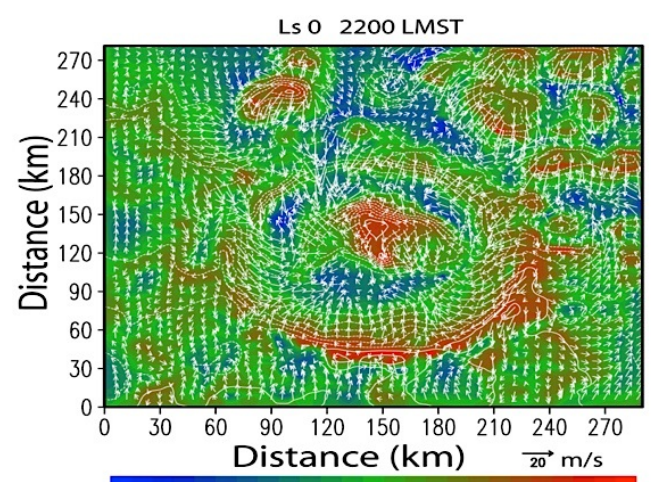

214218222226230234238242246250254258 Potential Temperature (K)

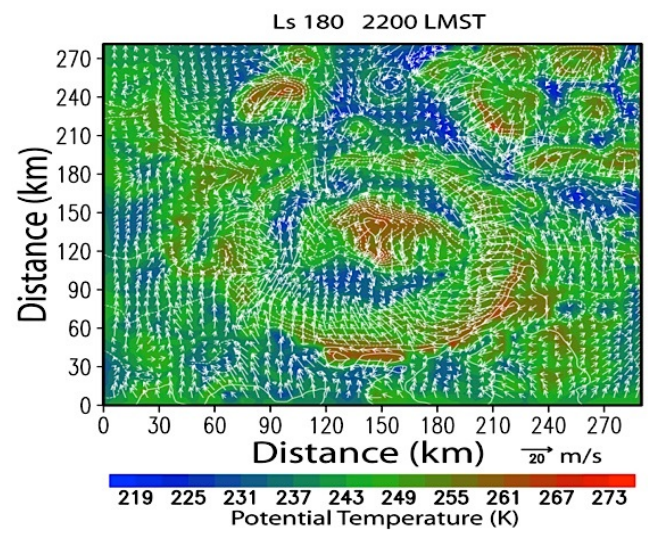

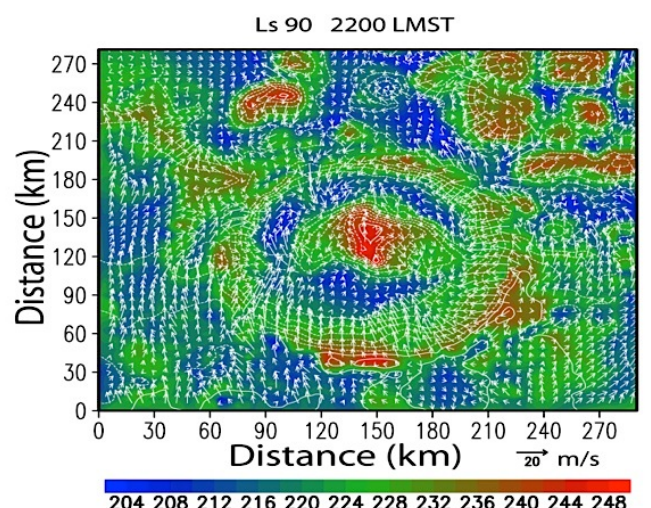

204208212216220224228232236240244248 Potential Temperature (K)

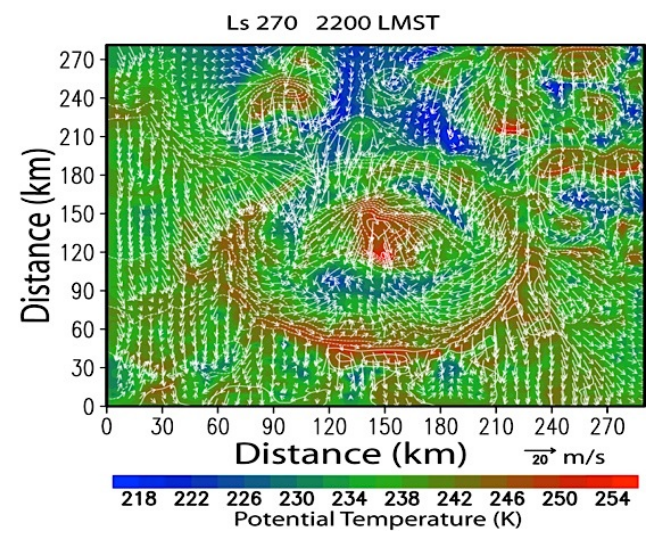

Figura 11. Igual que en figura 10 pero a las 2200 LMST.

En la Figura 12 se puede apreciar claramente como una fuerte corriente de aire frío penetra por el borde norte del cráter hacia las 2200 LMST en Ls 270. Este viento no es tan sólo aire fluyendo a través del accidente topográfico de Peace Vallis, sino más bien una inundación completa por todo el borde nornoroeste. En ese momento las masas de aire del interior del cráter están bien mezcladas con las del exterior debido a los vientos que lo inundan desde el norte. Algunos accidentes topográficos, como el antiguo cauce fluvial Peace Vallis al noroeste del cráter Gale, parecen proporcionar una vía con menor resistencia para el intercambio de aire entre el cráter y el exterior, pero ese intercambio parece ser mucho más limitado en Ls 0, 90 y 180. 


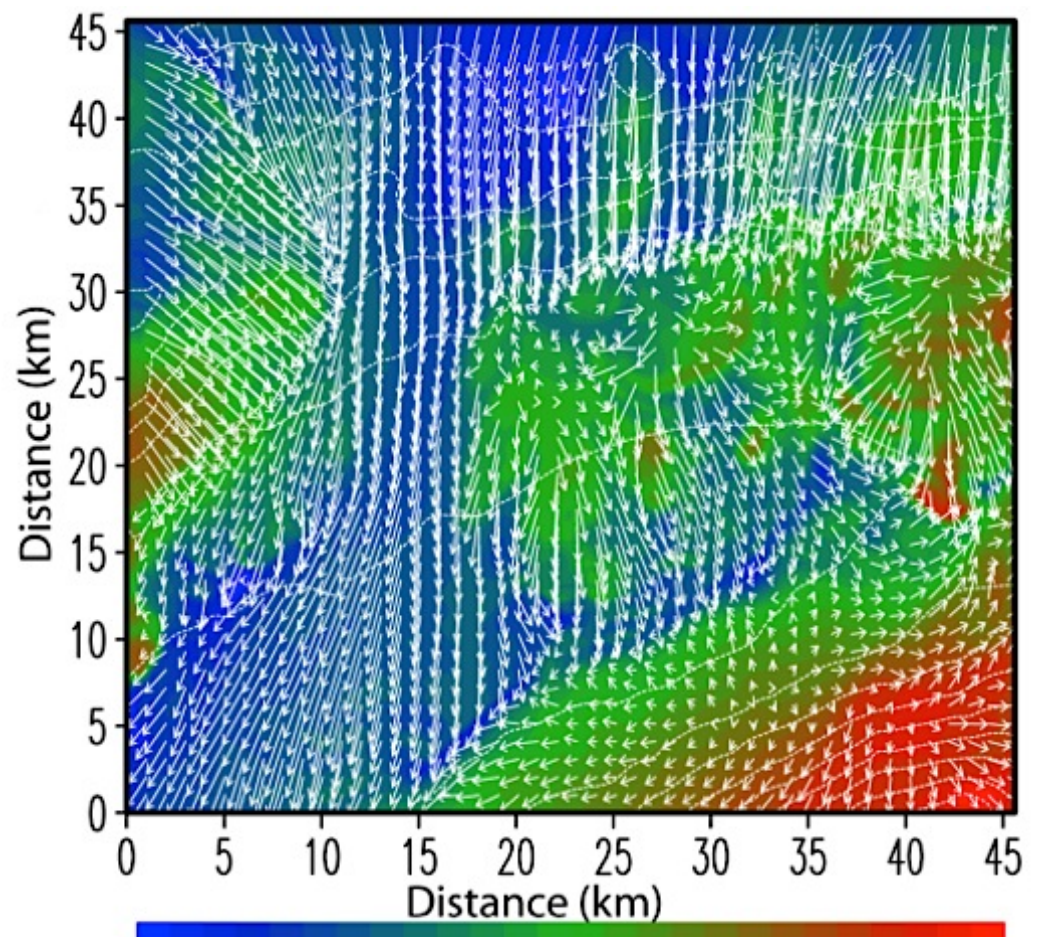

\section{9 Potential Temperature $(\mathrm{K}) \quad \overline{20} \mathrm{~m} / \mathrm{s}$}

Figura 12. Malla de mayor resolución en Ls 270 a las 2200LMST. Dirección y velocidad del viento en superficie (vectores) y temperatura potencial (colores) a las 2200 LMST. La escala del vector de viento se muestra en la parte inferior derecha de la imagen. Figura obtenida de Rafkin et al. 2016.

Los vientos también soplan hacia abajo en la dicotomía topográfica en todas las estaciones excepto en Ls 270, donde persisten los vientos del norte, ya que los vientos globales de componente norte consiguen derrotar por la noche a los vientos de componente sur descendentes por la dicotomía (Figura 13). 

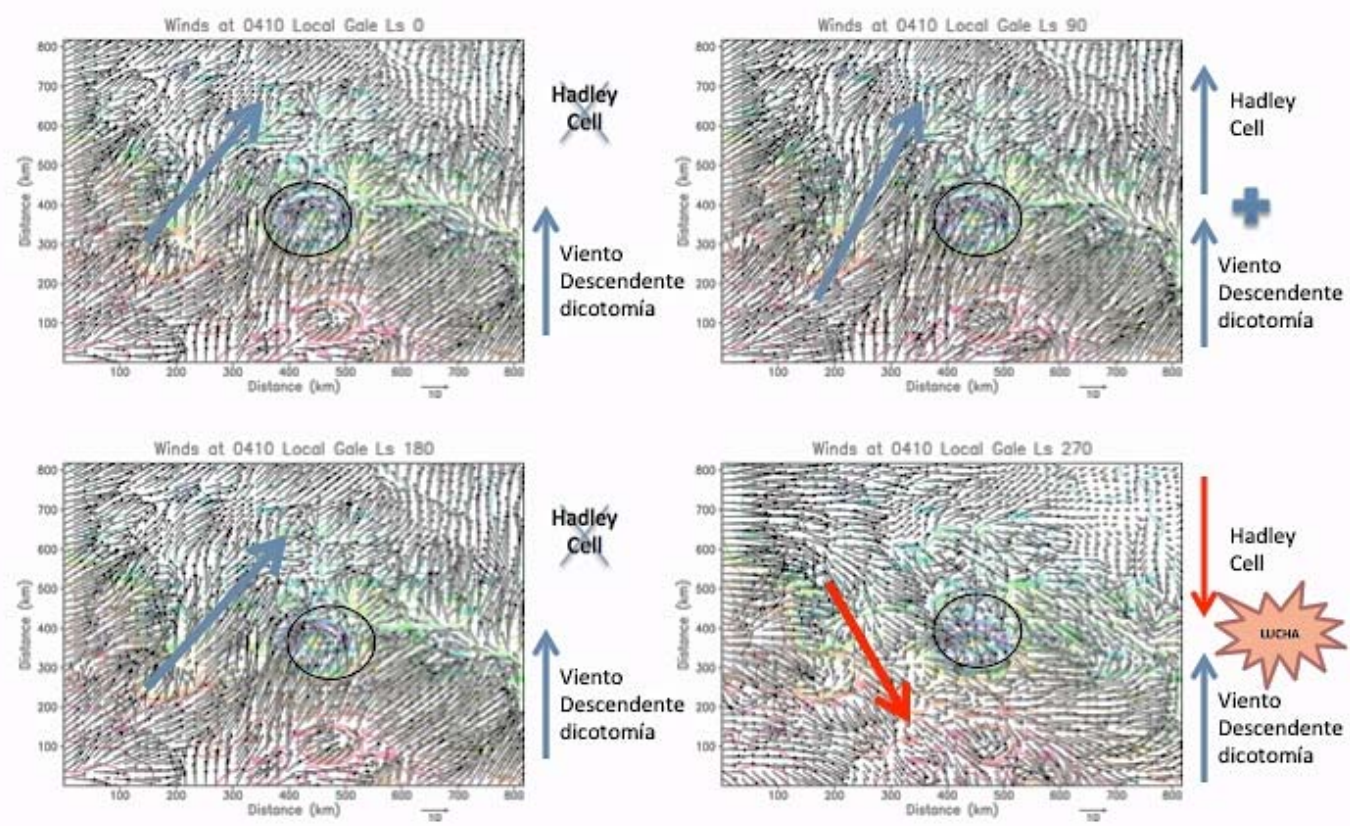

Figura 13. Escala regional durante la noche (malla 4, 9km res.) (0410 LMST). Fuerte influencia de la dicotomía y vientos regionales de componente sur en todas las estaciones excepto en Ls 270. Los vientos sinópticos de la célula de Hadley derrotan a los de la dicotomía en Ls 270.

\subsection{Vientos de ladera y ondas de montaña}

Los cortes en sección verticales proporcionan una clara representación de los vientos de ladera y de las ondas de montaña, cuando se estos producen. La Figura 14 muestra las secciones verticales de sur a norte a las 2200 LMST en función de la estación. En Ls 90 y Ls 180, no se muestra ninguna evidencia significativa de ondas de gravedad o de montaña. En Ls 0 aparece una onda en el borde norte del cráter, pero se encuentra muy por encima del suelo del mismo. Las ondas más fuertes se identifican claramente al norte del cráter en Ls 270 a las 2200 LMST, alcanzando la superficie y transportando el aire caliente en altura directamente al suelo, donde se mezcla con el aire frío, diluyéndolo.

Se cree que muchos de los vientos descendentes de ladera nocturnos que se producen en Marte son de origen catabático (Spiga et al., 2011). Los vientos catabáticos son generados por la aceleración gravitacional del aire frío impulsados hacia abajo por una pendiente. Pero en los vientos descendentes del cráter Gale hay algo más. Si bien es cierto que existen elementos de vientos catabáticos asociados a ondas de montaña especialmente justo después del atardecer, las ondas de montaña observadas son 
generadas dinámicamente y se deben principalmente a los vientos sinópticos interactuando con la topografía.
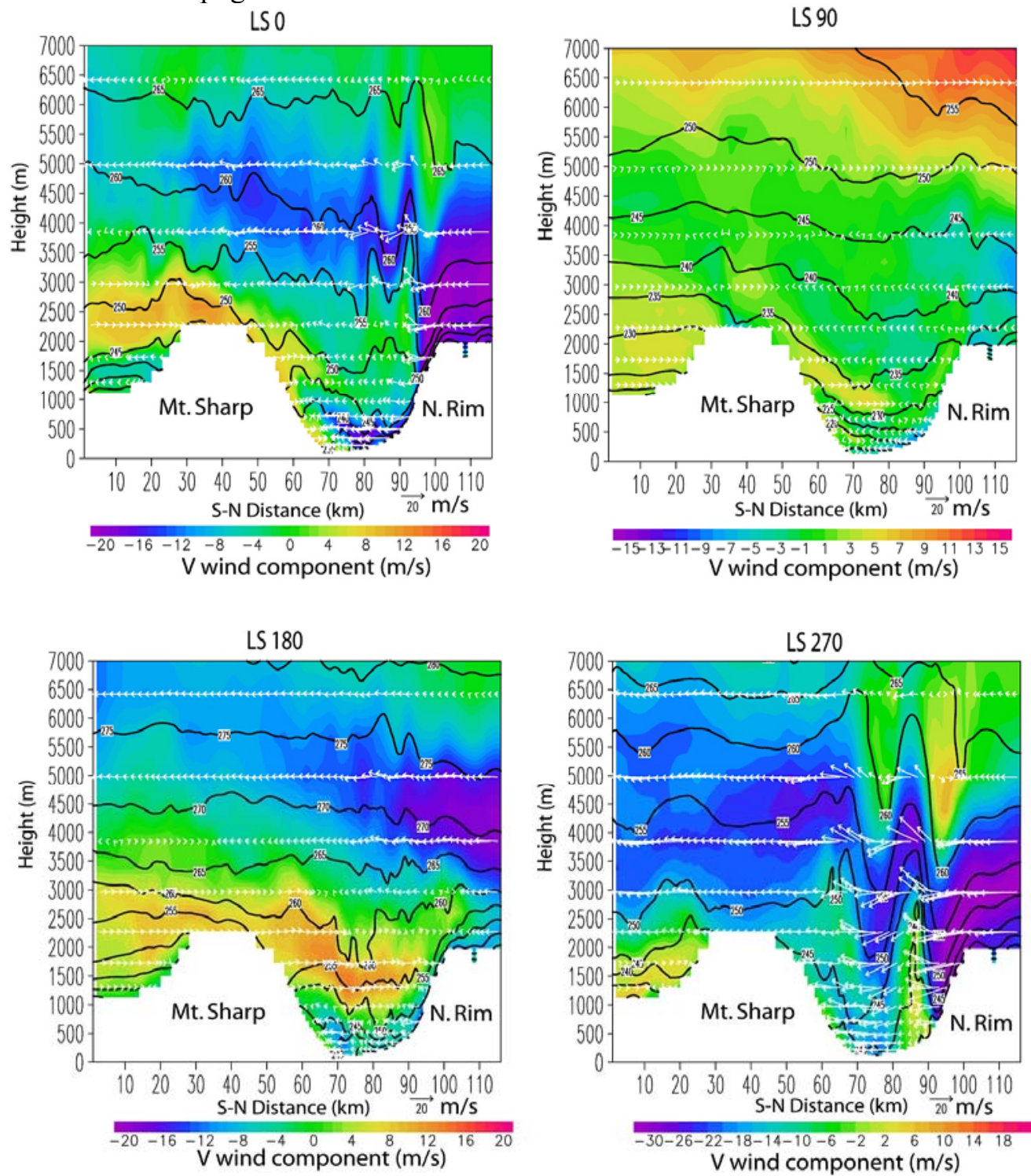

Figura 14. Corte en sección vertical (de sur a norte). Los vectores representan el viento en el plano de la imagen. Los colores representan la velocidad del viento horizontal y los contornos representan la temperatura potencial. Malla 6 en función de la estación a las 2200 LMST. Figura obtenida de Rafkin et al. 2016. 
En Ls 270, los vientos del norte son particularmente fuertes debido a la circulación meridional global. Sin embargo, el desarrollo de una capa de inversión térmica después del atardecer en los niveles más bajos, proporciona las condiciones ideales para la generación de ondas de montañas (sobre todo en los bordes y parte externa del cráter), que descienden hasta la superficie alrededor de las 2200 LMST. Al amanecer, la capa de inversión desaparece y el escenario favorable para la generación de ondas de montaña se disipa.

En Ls 0, 90 y 180, a medida que el aire frío desciende, se va encontrando a su paso con aire cada vez más frío (en el suelo del cráter la temperatura potencial es hasta $20 \mathrm{~K}$ más baja que el aire descendente). En ese momento, el aire descendente se desliza sobre el aire más frío, pasando directamente por encima del cráter sin interaccionar con las masas de aire que tiene en su interior, motivo por el cual se puede decir que el cráter se encuentra semiaislado la mayor parte del año.

\subsection{Capa Limite Atmosférica (CLA) debilitada}

La CLA es donde se producen los vórtices convectivos y los remolinos de polvo. Una capa límite poco profunda (distancia pequeña entre el suelo y la atmósfera libre) no favorece la formación de vórtices convectivos, por lo tanto no es sorprendente la ausencia en los datos de REMS de vórtices potentes y dust devils. En principio esperaríamos que las masas de aire en el exterior del cráter, donde los dust devils son más activos, fueran más polvorientas que las del interior del mismo. A medida que estas masas de aire se advectan sobre el cráter, se va formando una capa de polvo en altura. Curiosamente, el mayor número de vórtices de pequeña escala se produce entre las 1900 y las 2200 LMST en Ls 270 (Kahanpää et al., 2015). Estos vórtices no pueden ser convectivos, ya que la inestabilidad atmosférica no es producida por convección. En su lugar, los vórtices son generados por turbulencia de origen mecánico, probablemente ondas de montaña asociadas a rachas de viento. Además, no es sorprendente que la cantidad de remolinos de polvo sea tan pequeña, ya que los procesos de subsidencia, la CLA poco profunda y el gradiente adiabático estable (subadiabático), no favorecen la formación de dust devils.

En la Figura 15 se muestra un corte en sección vertical de sur a norte del cráter Gale a las 1600 LMST, para cada una de las estaciones simuladas con MRAMS. En ese momento, la CLA debe estar plenamente desarrollada y cerca de su profundidad máxima, sin embargo en el caso de Gale esta tiene solo una altura de 1-1.5 km, muy baja comparada con los 8-10 km que suele tener de media el planeta. Además, el flujo de ladera ascendente asociado a la dicotomía topográfica, que tarda más en desarrollarse que los flujos ascendentes por las laderas del cráter, debe estar completamente formado. Si observamos la temperatura potencial, se puede concluir que existe una fuerte inversión térmica justo encima del cráter en todas las estaciones. En la parte norte del cráter, la temperatura potencial aumenta con la altura, lo que significa que el perfil térmico vertical es estable en lugar de neutro. Con la excepción de Ls 270, cada 
estación muestra el patrón de pendiente ascendente local esperado, a pesar de que es alterada por circulaciones de mayor escala, como se ha mencionado previamente.
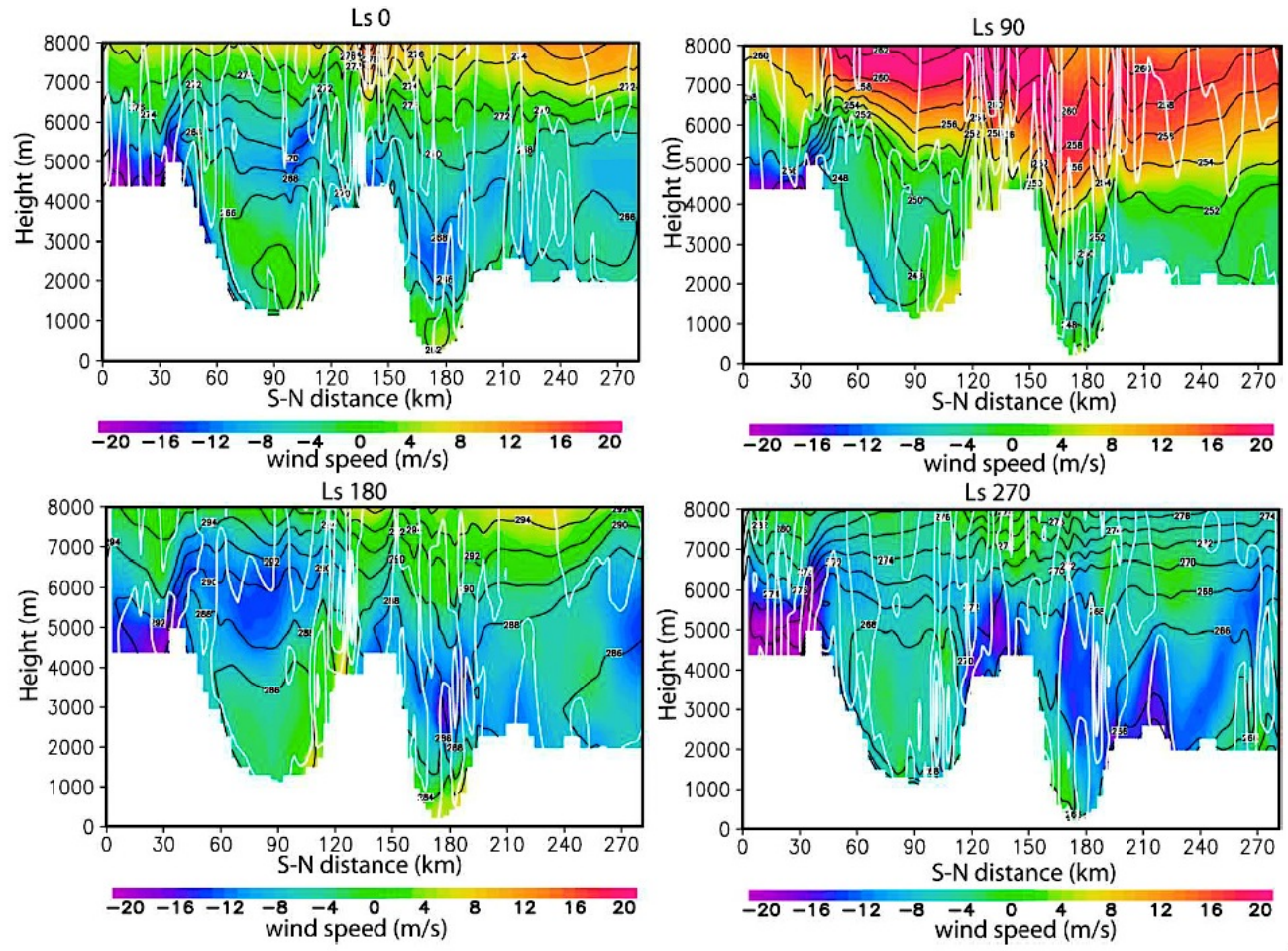

Figura 15. Corte en sección vertical de sur a norte a las 1600 LMST. Velocidad del viento horizontal en el plano de la sección Horizontal representado por escala de colores. La temperatura potencial y la velocidad vertical están representadas por contornos negros y blancos respectivamente. Los intervalos del contorno de la velocidad vertical son de $2 \mathrm{~m} / \mathrm{s}$. El plano del corte en sección es el oeste del monte Sharp, en $\mathrm{x} \approx 120 \mathrm{~km}$.

A diferencia de las circulaciones descendentes de ladera, las ascendentes parecen transportar eficientemente el aire del suelo del cráter hacia los bordes del mismo. Sin embargo, este aire generalmente cambia de sentido en altura y vuelve a descender en subsidencia. El movimiento hacia abajo actúa para debilitar la CLA. Sin duda, algo de aire se mezcla con la atmósfera de fuera del cráter, pero la mayor parte parece quedarse semiaislado dentro de él.

\section{Conclusiones}

El uso de los modelos de área limitada para simular la meteorología de Marte se ha convertido en una parte fundamental para la interpretación de los datos generados por 
las misiones marcianas y para proporcionar las restricciones de sus condiciones ambientales. Además son una herramienta fundamental para la evaluación de riesgos en la etapa de entrada, descenso y aterrizaje de las misiones al planeta rojo. Los procesos de mesoescala se producen en el dominio espacio-temporal comprendido entre la microescala y la escala sinóptica. Las circulaciones de mesoescala en Marte que contribuyen notablemente a la estructura y dinámica de la atmósfera son los vientos de ladera, los procesos de inyección de polvo atmosférico y los procesos de transporte de volátiles en la capa límite atmosférica. El modelo de mesoescala MRAMS se ha aplicado al cráter Gale, donde el rover Curiosity de la misión Mars Science Laboratory (MSL) de la NASA aterrizó en agosto de 2012 con la estación meteorológica española REMS a bordo. El modelo MRAMS se ha validado frente a los datos de REMS y los resultados de la comparación nos permiten concluir que el modelo representa las condiciones meteorológicas del cráter Gale de una forma fidedigna. Se aprecia gran similitud entre las simulaciones y las observaciones. La temperatura del suelo y la presión son los parámetros más robustos con los que validar las predicciones del modelo.

A diferencia de las anteriores misiones marcianas con instrumentación meteorológica, las cuales se encontraban en zonas llanas, la misión MSL se encuentra en una zona topográficamente muy compleja. Los datos obtenidos por el instrumento REMS en el cráter Gale revelan un entorno meteorológico dinámicamente muy complejo. Las circulaciones globales y regionales desempeñan un papel crucial en la meteorología del cráter. Comprender el contexto de las diferentes escalas (local, regional y global) y como interaccionan entre ellas es fundamental. Dentro del cráter se producen circulaciones atmosféricas, fruto de la suma no lineal de todas estas escalas. Los flujos atmosféricos locales son extremadamente complejos dentro del cráter, especialmente por la noche. Los mayores cambios estacionales se producen en Ls 270 . Los vientos descendientes provienen en todas las estaciones del hemisferio sur, excepto en Ls 270 en el que provienen del hemisferio norte. Los vientos más fuertes se producen en Ls 270 y sobre todo durante la noche debido a ondas de montaña. La masa de aire del cráter es generalmente más fría que la del exterior. Los vientos de ladera descendentes generalmente no se introducen en el fondo del cráter. Excepto en Ls 270, el flujo de aire suele pasar por encima del cráter, generando así una capa límite muy estable. Esta reducción en la mezcla de masas de aire debe tener una fuerte influencia sobre los ciclos del agua, polvo y gases dentro del cráter. Se concluye que la inyección de polvo atmosférico y la existencia de dust devils dentro del cráter son muy limitadas. El aire que sobrevuela el cráter tiene mayor contenido en polvo. Se aprecian variaciones de temperatura por la noche debido a corrientes de densidad complejas y ondas de gravedad. Se aprecia turbulencia nocturna probablemente debido a inestabilidades por vientos de cizalla o a vientos de ladera descendentes. Tanto en el modelo como en las observaciones se aprecian diferentes eventos dinámicos: ondas gravitatorias y ondas de montaña. Los resultados del modelo deben 
considerarse representativos, pero no necesariamente como predicciones de la hora o lugar exactos de los eventos meteorológicos.

\section{Agradecimientos}

Los autores agradecen encarecidamente la invitación a escribir este artículo de los editores Francisco Valero, Miguel Herraiz Sarachaga y Jesús Martínez-Frías y los didácticos comentarios de Daniel Santos Muñoz. Los autores a su vez están muy agradecidos por el gran trabajo realizado por todo el equipo de MSL y, muy especialmente, por el esfuerzo de los equipos de ingeniería y ciencia de REMS, cuya dedicación ha permitido que este artículo haya podido elaborarse. Este trabajo ha sido financiado por el Ministerio de Economía y Competitividad (contratos AYA201125720 y AYA2012-38707) y por el Jet Propulsion Laboratory de NASA (contrato 1509303).

\section{Referencias}

(2002), Simulation of surface meteorology at the Pathfinder and VL1 sites using a Mars mesoscale model, Journal of Geophysical Research: Planets, 107, 4. BASU, S., RICHARDSON M. I., WILSON R. J. (2004), Simulation of the Martian dust cycle with the GFDL Mars GCM. Journal of Geophysical Research: Planets 109, 11.

CANTOR, B. A., JAMES P. B., CAPLINGER M., WOLFF M. J. (2001), Martian dust storms: 1999 Mars Orbiter Camera observations, Journal of Geophysical Research: Planets, 106, 10, 23653-23687.

CHEN, A., et al. (2010), Atmospheric risk assessment for the Mars Science Laboratory entry, descent, and landing system: Aerospace Conference, 2010 IEEE.

CONRATH, B. J. (1975), Thermal structure of the Martian atmosphere during the dissipation of the dust storm of 1971. Icarus 24, 1, 36-46.

FISHER, J. A., RICHARDSON M. I, NEWMAN C. E., SZWAST M. A., GRAF C., BASU S., EWALD S. P., TOIGO A. D., WILSON R. J. (2005), A survey of Martian dust devil activity using Mars Global Surveyor Mars Orbiter Camera images, Journal of Geophysical Research: Planets, 110, 3.

GIERASCH, P., GOODY R. (1968), A study of the thermal and dynamical structure of the martian lower atmosphere, Planet Space Sci.,16, 5, 615-646.

GÓMEZ-ELVIRA, J., et al. (2012), REMS: The environmental sensor suite for the Mars Science Laboratory rover, Space Sci. Rev., 170, 583-640.

HABERLE, R. M., et al. (1993), A boundary-layer model for Mars: Comparison with Viking lander and entry data. Journal of the Atmospheric Sciences 50, 11, 15441559 . 
HABERLE, R. M. (1993), Mars atmospheric dynamics as simulated by the NASA/Ames general circulation model, Journal of Geophysical Research, 98, 3093-3124.

HABERLE, R.M., LEOVY C. B., POLLACK J. B. (1982), Some effects of global dust storms on the atmospheric circulation of Mars, Icarus, 50, 322-367.

HAMILTON, V. E. et al. (2014), Observations and preliminary science results from the first 100 sols of MSL Rover Environmental Monitoring Station ground temperature sensor measurements at Gale Crater. Journal of Geophysical Research: Planets 119, 4, 745-770.

HARRI, A. M., et al. (2014), Pressure observations by the Curiosity rover: Initial results. Journal of Geophysical Research: Planets 119, 1, 82-92.

KAHRE, M. A., J. R. MURPHY, HABERLE R. M. (2006), Modelling the Martian dust cycle and surface dust reservoirs with the NASA Ames general circulation model, Journal of Geophysical Research: Planets, 111, 6.

LARSEN, S. E., JØRGENSEN H. E., LANDBERG L., TILLMAN J. E. (2002), Aspects of the atmospheric surface layers on Mars and Earth. Boundary-Layer Meteorology 105, 3, 451-470.

MARTÍNEZ G., DE LA TORRE M., VICENTE-RETORTILLO A., KEMPPINEN O., RENNO N., LEMMON M. (2016), An Overview of the Environmental Conditions at Gale Crater from MSL/REMS Measurements. Revista Española de Física de la Tierra (this issue).

MICHAELS, T. I., RAFKIN S. (2004), Large-eddy simulation of atmospheric convection on Mars, Quarterly Journal of the Royal Meteorological Society, 130, 599, 1251-1274.

MICHAELS, T. I., COLAPRETE A., RAFKIN S. (2006), Significant vertical water transport by mountain-induced circulations on Mars, Geophysical Research Letters, 33, 16, $5 \mathrm{pp}$.

NEWMAN, C. E., LEWIS S. R., READ P. L., FORGET F. (2002), Modeling the Martian dust cycle, 1. Representations of dust transport processes. Journal of Geophysical Research: Planets, 107, 12.

PIELKE, R. A., et al. (1992), A comprehensive meteorological modeling systemRAMS, Meteorology and Atmospheric Physics, 49, 1, 69-91.

PLA-GARCÍA J., RAFKIN S., KAHRE M., GÓMEZ-ELVIRA J., HAMILTON V., MARÍN M., NAVARRO S., TORRES J., VASAVADA A. (2016), The meteorology of Gale Crater as determined from Rover Environmental Monitoring Station observations and numerical modeling. Part I: Interpretation. Icarus, 280, 103-113

RAFKIN, S., PLA-GARCÍA J., KAHRE M., GÓMEZ-ELVIRA J., HAMILTON V., MARÍN M., NAVARRO S., TORRES J., VASAVADA A. (2016), The meteorology of Gale Crater as determined from Rover Environmental Monitoring Station observations and numerical modeling. Part II: Interpretation. Icarus, 280, 114-138 
RAFKIN, S. (2011), The Potential Importance of Non-Local, Deep Transport on the Energetics, Momentum, Chemistry, and Aerosol Distributions in the Atmospheres of Earth, Mars, and Titan, Planet. Space Sci. 60, 147-154

RAFKIN, S., MICHAELS T. I. (2004), Meteorological predictions for the Beagle 2 mission to Mars. Geophysical Research Letters, 31, 1.

RAFKIN, S., MICHAELS T. I. (2003), Meteorological predictions for 2003 Mars Exploration Rover high-priority landing sites, Journal of Geophysical Research, $108,12,8091$.

RAFKIN, S. C. R., STA. MARIA M. R. V. , MICHAELS T. I. (2002): Simulation of the atmospheric thermal circulation of a martian volcano using a mesoscale numerical model. Nature, 419, 697-699.

RAFKIN, S., HABERLE R. M., MICHAELS T. I. (2001), The Mars Regional Atmospheric Modeling System: Model Description and Selected Simulations, Icarus, 151, 2, 228-256.

SAVIJÄRVI, H., KAUHANEN J. (2008), Surface and boundary-layer modelling for the Mars Exploration Rover sites. Quarterly Journal of the Royal Meteorological Society 134, 632, 635-641.

SMITH, M. D., et al. (2004), First Atmospheric Science Results from the Mars Exploration Rovers Mini-TES, Science, 306, 5702, 1750-1753.

SCHOFIELD, J.T., BARNES, J.R., CRISP, D. et al., (1997). The Mars pathfinder atmospheric structure investigation/meteorology (ASI/MET) experiment. Science $278,5344,1752-1758$.

SPANOVICH N., SMITH M.D., SMITH P.H., WOLFF M.J., CHRISTENSEN P.R., SQUYRES S.W. (2006), Surface and near-surface atmospheric temperatures for the Mars Exploration Rover landing sites. Icarus.

SPIGA, A., FORGET F., MADELEINE J. B., MONTABONE L., LEWIS S. R., MILLOUR E. (2011), The impact of martian mesoscale winds on surface temperature and on the determination of thermal inertia, Icarus, 212, 2, 504-519.

SPIGA, A., FORGET F., MADELEINE J. B., MONTABONE L., LEWIS S. R., MILLOUR E. (2011), Elements of comparison between Martian and terrestrial mesoscale meteorological phenomena: Katabatic winds and boundary layer convection, Planet Space Sci., 59, 10, 915-922.

TAMPPARI, L.K., BARNES, J., BONFIGLIO, E., CANTOR, B., FRIEDSON, A.J., GHOSH, A., GROVER, M.R., KASS, D., MARTIN, T.Z., MELLON, M., MICHAELS, T., 2008. Expected atmospheric environment for the Phoenix landing season and location. Journal of Geophysical Research: Planets, 113, 3.

TOIGO, A. D., RICHARDSON M. I. (2003), Meteorology of proposed Mars Exploration Rover landing sites. Journal of Geophysical Research: Planets 108, 12.

TYLER, D., BARNES J. R. (2005), A mesoscale model study of summertime atmospheric circulations in the north polar region of Mars, Journal of Geophysical Research: Planets 110,6 . 
TYLER, D., BARNES J. R., HABERLE R. M. VASAVADA A. et al. (2012), Assessment of Environments for Mars Science Laboratory Entry, Descent, and Surface Operations. Space Science Review 170, 1, 793-835

YE, Z. J., M. SEGAL, R. A. PIELKE (1990), A comparative study of daytime thermally induced upslope flow on Mars and Earth. Journal of the Atmospheric Sciences $47,5,612-628$. 